Preprint typeset in JHEP style - HYPER VERSION

ROME1/1453/07

UB-ECM-PF-07-16

December 2007

\title{
Charm-quark fragmentation with an effective coupling constant
}

\author{
Gennaro Corcella \\ Dipartimento di Fisica, Università di Roma 'La Sapienza' \\ P.le A. Moro 2, I-00185 Roma, Italy. \\ E-mail: Gennaro.Corcella@roma1.infn.it \\ Giancarlo Ferrera \\ Departament d'Estructura i Constituents de la Matèria, \\ Universitat de Barcelona, \\ Diagonal 647, E-08028 Barcelona, Spain \\ E-mail: ferrera@ecm.ub.es
}

\begin{abstract}
We use a recently proposed non-perturbative model, based on an effective strong coupling constant and free from tunable parameters, to study $c$-flavoured hadron production in $e^{+} e^{-}$annihilation. Charm-quark production is described in the framework of perturbative fragmentation functions, with NLO coefficient functions, NLL non-singlet DGLAP evolution and NNLL large- $x$ resummation. We model hadronization effects by means of the effective coupling constant in the NNLO approximation and compare our results with experimental data taken at the $Z^{0}$ pole and at the $\Upsilon(4 S)$ resonance. We find that, within the experimental and theoretical uncertainties, our model is able to give a reasonable description of $D^{*+}$-meson spectra from ALEPH for $x<1-\Lambda / m_{c}$. More serious discrepancies are instead present when comparing with $D$ and $D^{*}$ data from BELLE and CLEO in $x$-space. Within the errors, our model is nonetheless capable of reproducing the first ten Mellin moments of all considered data sets. However, the fairly large theoretical uncertainties call for a full NNLO/NNLL analysis.
\end{abstract}

KeYwords: QCD, Heavy Quark Physics, NLO Computations. 


\section{Contents}

1. Introduction 1

2. Charm-quark production 2

2.1 Perturbative fragmentation functions 2

2.2 Large- $x$ resummation 3

3. Effective coupling constant

4. Results in $x$-space 10

4.1 Comparison with ALEPH data 11

4.2 Comparison with CLEO and BELLE data 14

5. Results in $N$-space 17

6. Conclusions 21

\section{Introduction}

The hadronization of partons into hadrons, for the time being, cannot be calculated from first principles, but it is usually described in terms of phenomenological models, such as the Kartvelishvili [1] or Peterson [2] non-perturbative fragmentation functions, containing few parameters which need to be tuned to experimental data. It was recently proposed [3, 国, however, a non-perturbative model, based on the work in refs. [5, 6], including power corrections via an effective strong coupling constant, which does not exhibit the Landau pole any longer and includes absorptive effects due to gluon branching. The interesting feature of such a model is that it does not contain any extra free parameter to be fitted to the data, besides the ones entering in the parton-level calculation. In [3], such a model was used in the framework of $B$-meson decays and it was found good agreement with the data on the photon spectrum and on the hadron-mass distribution in radiative and semileptonic decays, respectively. In 淊 the effective coupling was employed in the framework of bottomquark fragmentation and, within the theoretical uncertainties, a reasonable fit of LEP and SLD data on $B$-hadron spectra was obtained in both $x$ and moment spaces.

Although the results in [3, 4] are encouraging, it is nonetheless mandatory to consider more data and observables to validate the effective-coupling model. In this paper, we consider charm-quark fragmentation in $e^{+} e^{-}$processes and investigate how our nonperturbative model fares against $D$-meson data from LEP and $B$-factories. In fact, charm 
production involves pretty different scales with respect to $b$-quark fragmentation, and therefore the comparison with $D$-hadron spectra should help to shed light on our model. Considering charm production at the $Z^{0}$ pole and at the $\Upsilon(4 S)$ resonance, furthermore, is also interesting to understand how our model behaves when the process hard scale changes. Perturbative charm production will be described in the framework of perturbative fragmentation functions [7], using the same approximations carried out in [ [4], and the effective coupling constant will be our only source of non-perturbative power corrections.

The plan of the present paper is the following. In section 2 we shall review the main points of the parton-level computation, based on the perturbative fragmentation formalism, and including large- $x$ resummation in both coefficient function and initial condition of the perturbative fragmentation function. In section 3 we shall discuss the effective coupling constant and the inclusion of non-perturbative corrections to charm-quark fragmentation. In section 4 we shall compare the results with charmed-meson spectra from LEP and $B$ factories in $x$-space, whereas we present our analysis in Mellin moment space in section 5 . We shall finally summarize our main results in section 6 .

\section{Charm-quark production}

In this section we shall discuss our calculation for charm-quark production. For the sake of consistency, and given the tight relation between perturbative calculation and nonperturbative corrections, our computation will be carried out along the lines of ref. 44. Therefore, we shall just point out the main issues involved in the calculation and refer to [4] for further details.

\subsection{Perturbative fragmentation functions}

We consider $c \bar{c}$-pair production in $e^{+} e^{-}$annihilation at next-to-leading order (NLO) in the strong coupling constant $\alpha_{S}$ :

$$
e^{+} e^{-} \rightarrow P(Q) \rightarrow c\left(p_{c}\right) \bar{c}\left(p_{\bar{c}}\right)\left(g\left(p_{g}\right)\right)
$$

and define the charm-quark energy fraction:

$$
x=\frac{2 p_{c} \cdot Q}{Q^{2}} .
$$

In the following, we shall consider charm production at LEP, where $P$ is a $Z^{0}$ boson and $Q=m_{Z}$, as well as $c$-quark fragmentation at the $\Upsilon(4 S)$ resonance, i.e. $Q=m_{\Upsilon(4 S)}$ and the $c \bar{c}$ pair coming from the decay of a virtual photon $\left(P=\gamma^{*}\right)$. In principle, in $e^{+} e^{-}$annihilation charm quarks can also come from other processes, such as the decay of bottomed hadrons produced via $Z^{0}(\Upsilon(4 S)) \rightarrow b \bar{b}$. However, as we shall discuss in detail in section 4 , our analysis will only deal with direct $c \bar{c}$ production.

The perturbative fragmentation approach [0], up to power corrections, factorizes the energy distribution of a heavy quark, the charm quark in our case, as the convolution of a coefficient function, associated with the emission off a massless parton, and a perturbative 
fragmentation function, expressing the transition of the light parton into a heavy quark. This way, the $c$-quark spectrum reads:

$$
\begin{aligned}
\frac{1}{\sigma} \frac{d \sigma}{d x}\left(x, Q, m_{c}\right) & =\sum_{i} \int_{x}^{1} \frac{d z}{z}\left[\frac{1}{\sigma} \frac{d \hat{\sigma}_{i}}{d z}\left(z, Q, \mu_{R}, \mu_{F}\right)\right]^{\overline{\mathrm{MS}}} D_{i}^{\overline{\mathrm{MS}}}\left(\frac{x}{z}, \mu_{F}, m_{c}\right) \\
& +\mathcal{O}\left(\left(m_{c} / Q\right)^{p}\right) .
\end{aligned}
$$

In eq. (2.3), $p \geq 1, d \hat{\sigma}_{i} / d z$ is the differential cross section for the production of a massless parton $i$ after subtracting the collinear singularity in the $\overline{\mathrm{MS}}$ factorization scheme; $\mu_{R}$ and $\mu_{F}$ are the renormalization and factorization scales; $\sigma$ is the NLO $e^{+} e^{-} \rightarrow q \bar{q}(g)$ cross section. Hereafter, we shall neglect charm production via $g \rightarrow c \bar{c}$ splitting. In fact, we can anticipate that, when comparing with data, secondary $c \bar{c}$ production will be either subtracted off the sample which we shall analyse or negligible at the centre-ofmass considered energy. This implies that $i=c$ in eq. (2.3) and $D_{c}^{\overline{\mathrm{MS}}}$ is the perturbative fragmentation function expressing the fragmentation of a massless $c$ into a massive $c$. The NLO $\overline{\mathrm{MS}}$ coefficient function for $e^{+} e^{-} \rightarrow q \bar{q}$ processes can be found in [8].

The perturbative fragmentation function follows the DGLAP evolution equations [9, 10]; its value at a any scale $\mu_{F}$ can be obtained once an initial condition at $\mu_{0 F}$ is given. In [7] the initial condition $D_{c}^{\mathrm{ini}}\left(x, \mu_{0 F}, m_{c}\right)$ was calculated in the NLO approximation and its process-independence was established on more general grounds in [11. It is given by:

$D_{c}^{\mathrm{ini}}\left(x, \alpha_{S}\left(\mu_{0 R}^{2}\right), \mu_{0 F}^{2}, m_{c}^{2}\right)=\delta(1-x)+\frac{\alpha_{S}\left(\mu_{0 R}^{2}\right) C_{F}}{2 \pi}\left[\frac{1+x^{2}}{1-x}\left(\ln \frac{\mu_{0 F}^{2}}{m_{c}^{2}}-2 \ln (1-x)-1\right)\right]_{+}$.

As discussed in [7], solving the DGLAP equations for an evolution from $\mu_{0 F}$ to $\mu_{F}$, with a NLO kernel, allows one to resum leading (LL) $\alpha_{S}^{n} \ln ^{n}\left(\mu_{F}^{2} / \mu_{0 F}^{2}\right)$ and next-to-leading (NLL) $\alpha_{S}^{n} \ln ^{n-1}\left(\mu_{F}^{2} / \mu_{0 F}^{2}\right)$ logarithms. Setting $\mu_{0 F} \simeq m_{c}$ and $\mu_{F} \simeq Q$, one resums the large $\ln \left(Q^{2} / m_{c}^{2}\right)$ appearing in the massive NLO spectrum [7]. The resummation of such mass logarithms is usually called collinear resummation. For the sake of working in the same perturbative framework as in «4, in the following we shall consider NLO coefficient functions and initial condition, along with NLL non-singlet DGLAP evolution. However, one could go beyond such a level of accuracy and include NNLO corrections to the coefficient function [12, 13, initial condition 114 and to the non-singlet splitting functions 15 entering in the kernel of the DGLAP equations. The gluon-initiated contribution to the initial condition, necessary to possibly extend the analysis to the singlet sector, was calculated in [7] and 16] to NLO and NNLO, respectively.

\subsection{Large- $x$ resummation}

Both coefficient function [7] and initial condition (2.4) contain terms, $\sim 1 /(1-x)_{+}$and $\sim[\ln (1-x) /(1-x)]_{+}$, enhanced when $x$ approaches 1 , which corresponds to soft- or collinear-gluon radiation. One needs to resum such contributions to all orders to improve the perturbative prediction (threshold resummation). As in 四], we shall implement threshold resummation, which is process-dependent in the coefficient function and processindependent in the initial condition [11], in the next-to-next-to-leading logarithmic (NNLL) 
approximation, following the general method of [17, 18]. Large- $x$ resummation is typically performed in Mellin moment-space, where the Mellin transform of the differential cross section reads:

$$
\sigma_{N}=\int_{0}^{1} d x x^{N-1} \frac{1}{\sigma} \frac{d \sigma}{d x} .
$$

In $N$-space, the enhanced contributions $\sim \alpha_{S} /(1-x)_{+}$and $\alpha_{S}[\ln (1-x) /(1-x)]_{+}$correspond to single $\left(\sim \alpha_{S} \ln N\right)$ and double $\left(\sim \alpha_{S} \ln ^{2} N\right)$ logarithms of the Mellin variable $N$. The resummed coefficient function is given by the following generalized exponential function [1]:

$$
\Delta_{N}^{(C)}\left[\alpha_{S}\left(\mu_{R}^{2}\right), \mu_{R}^{2}, \mu_{F}^{2}, Q^{2}\right]=\exp \left\{G_{N}^{(C)}\left[\alpha_{S}\left(\mu_{R}^{2}\right), \mu_{R}^{2}, \mu_{F}^{2}, Q^{2}\right]\right\}
$$

where

$$
\begin{aligned}
G_{N}^{(C)}\left[\alpha_{S}\left(\mu_{R}^{2}\right), \mu_{R}^{2}, \mu_{F}^{2}, Q^{2}\right] & =\int_{0}^{1} d z \frac{z^{N-1}-1}{1-z}\left\{\int_{\mu_{F}^{2}}^{Q^{2}(1-z)} \frac{d k^{2}}{k^{2}} A\left[\alpha_{S}\left(k^{2}\right)\right]\right. \\
& \left.+B\left[\alpha_{S}\left(Q^{2}(1-z)\right)\right]\right\}
\end{aligned}
$$

The exponent $G_{N}^{(C)}\left[\alpha_{S}\left(\mu_{R}^{2}\right), \mu_{R}^{2}, \mu_{F}^{2}, Q^{2}\right]$ resums the large logarithms of the Mellin variable; in the NNLL approximation, one keeps in the exponent terms $\sim \alpha_{S}^{n} \ln ^{n+1} N(\mathrm{LL}), \sim$ $\alpha_{S}^{n} \ln ^{n} N$ (NLL) and $\sim \alpha_{S}^{n} \ln ^{n-1} N$ (NNLL). As in [18], the integration variables are $z=$ $1-x_{g}, x_{g}$ being the gluon energy fraction, and $k^{2}=\left(p_{c}+p_{g}\right)^{2}(1-z)$. In soft approximation, $z \simeq x$; for small-angle radiation $k^{2} \simeq k_{\perp}^{2}$, the gluon transverse momentum with respect to the $c$.

In (2.7), function $A\left(\alpha_{S}\right)$ resums soft and collinear radiation, while $B\left(\alpha_{S}\right)$ includes all-order collinear and hard emissions. They can be expanded as a series in $\alpha_{S}$ as:

$$
\begin{aligned}
& A\left(\alpha_{S}\right)=\sum_{n=1}^{\infty}\left(\frac{\alpha_{S}}{\pi}\right)^{n} A^{(n)}, \\
& B\left(\alpha_{S}\right)=\sum_{n=1}^{\infty}\left(\frac{\alpha_{S}}{\pi}\right)^{n} B^{(n)} .
\end{aligned}
$$

In the NLL approximation, one needs to include the first two coefficients of $A\left(\alpha_{S}\right)$ and the first of $B\left(\alpha_{S}\right)$; to NNLL accuracy, $A^{(3)}$ and $B^{(2)}$ are also needed. The coefficients $A^{(1)}, A^{(2)}$ and $B^{(2)}$ can be found in [18]; more recent is the calculation of the NNLL contributions $A^{(3)}$ [19] and $B^{(2)}$ 20].

Likewise, the threshold-resummed initial condition reads [11]:

$$
\Delta_{N}^{(D)}\left[\alpha_{S}\left(\mu_{0 R}^{2}\right), \mu_{0 R}^{2}, \mu_{0 F}^{2}, m_{c}^{2}\right]=\exp \left\{G_{N}^{(D)}\left[\alpha_{S}\left(\mu_{0 R}^{2}\right), \mu_{0 R}^{2}, \mu_{0 F}^{2}, m_{c}^{2}\right]\right\},
$$

where

$$
\begin{aligned}
G_{N}^{(D)}\left[\alpha_{S}\left(\mu_{0 R}^{2}\right), \mu_{0 R}^{2}, \mu_{0 F}^{2}, m_{c}^{2}\right] & =\int_{0}^{1} d z \frac{z^{N-1}-1}{1-z}\left\{\int_{m_{c}^{2}(1-z)^{2}}^{\mu_{0 F}^{2}} \frac{d k^{2}}{k^{2}} A\left[\alpha_{S}\left(k^{2}\right)\right]\right. \\
& \left.+D\left[\alpha_{S}\left(m_{c}^{2}(1-z)^{2}\right)\right]\right\}
\end{aligned}
$$


with $k^{2}$ and $z$ defined as in (2.7). To NNLL accuracy, we need $A^{(1)}, A^{(2)}$ and $A^{(3)}$ and the first two coefficients of

$$
D\left(\alpha_{S}\right)=\sum_{n=1}^{\infty}\left(\frac{\alpha_{S}}{\pi}\right)^{n} D^{(n)},
$$

namely $D^{(1)}$ and $D^{(2)}$. Function $D\left(\alpha_{S}\right)$, called $H\left(\alpha_{S}\right)$ in [1], is characteristic of the fragmentation of heavy quarks and resums soft and large-angle radiation. Its $\mathcal{O}\left(\alpha_{S}\right)$ coefficient can be found in [11], while $D^{(2)}$ can be read from the formulas in [21, 22, 14]. In any case, all relevant NNLL threshold-resummation coefficients are reported in [4].

In the phenomenological analysis of [- 1 , the inclusion of NNLL effects, and especially the contribution $\sim \alpha_{S}^{3} A^{(3)}$ to function $A\left(\alpha_{S}\right)$, turned out to be necessary to reproduce the $b$-fragmentation data. In fact, as we shall point out in the next section, when using the effective coupling constant we need to redefine the threshold-resummation coefficients from the third order on. This way, it turns out that $A^{(3)}$ gets enhanced. The inclusion of NNLL terms in the resummed exponents shifted the $B$-hadron spectrum towards lower $x_{B}$ values and played a crucial role to obtain a reasonable description of LEP and SLD data (see figure 4 in ref. 四).

As in [3, 过, the Mellin transforms of our resummed expressions will be performed exactly and not according to the step-function approximation, which was instead employed in the resummations carried out in refs. [11, 18] ${ }^{1}$. In fact, as we shall discuss later, we will model non-perturbative effects to charm fragmentation by means of an effective coupling constant and it was found in 26] that the step-function approximation would suppress most power corrections included in the physical observables via the analytic coupling. In any case, as thoroughly detailed in [4], the issue of the power corrections which are transferred to the cross section by the effective coupling, and whether it is a better approximation performing the Mellin transforms in an exact or approximated way is currently an open issue and we cannot draw any firm conclusion. A careful analysis, along the lines of [27], will be anyway very welcome to clarify this point. For the time being, the exactness of the Mellin transforms should be seen as part of our non-perturbative model. We just point out that, unlike refs. [11, 18], where only logarithms of $N$ are resummed, in our approach even some constants and power-suppressed $\mathcal{O}(1 / N)$ terms are included in the exponents (2.6) and (2.10) thanks to the exact Mellin transforms. This implies that, any time we improve the accuracy of the large- $x$ resummation, e.g., from NLL to NNLL, we include in the resummed exponent not only subleading logarithms of $N$, but also constants and power corrections. This tight relation between perturbative and non-perturbative corrections is indeed a peculiar feature of our effective-coupling model.

As in 沟, the resummed results are matched to the exact NLO coefficient function and

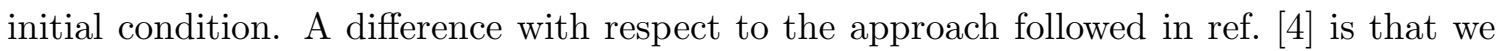
implement the so-called $\ln R$-matching [23, 24], corresponding to matching the logarithms of resummed and NLO expressions. We briefly review this matching strategy and how it

\footnotetext{
${ }^{1}$ In [11, 18], the longitudinal-momentum integration is done after performing the replacement $x^{N} \rightarrow$ $1-\Theta\left(1-z-\frac{e^{-\gamma_{E}}}{N}\right)$, which is a correct approximation to NLL accuracy. Beyond NLL, it can be generalized following the prescription presented in 25].
} 
compares with the standard method implemented in «. Referring, e.g., to the coefficient function, matched to the exact NLO one, it can be written (see eq. (4.2) in ref. [4) as:

$$
\begin{aligned}
C_{N}^{\prime \text { res }}\left[\alpha_{S}\left(\mu_{R}^{2}\right), \mu_{R}^{2}, \mu_{F}^{2}, Q^{2}\right] & =K^{(C)}\left[\alpha_{S}\left(\mu_{R}^{2}\right), \mu_{R}^{2}, \mu_{F}^{2}, Q^{2}\right] \Delta_{N}^{(C)}\left[\alpha_{S}\left(\mu_{R}^{2}\right), \mu_{R}^{2}, \mu_{F}^{2}, Q^{2}\right] \\
& +d_{N}^{(C)}\left[\alpha_{S}\left(\mu_{R}^{2}\right), \mu_{R}^{2}, \mu_{F}^{2}, Q^{2}\right] .
\end{aligned}
$$

In (2.13), $\Delta_{N}^{(C)}$ is the resummed coefficient function, given in eq. (2.6),

$$
K^{(C)}\left[\alpha_{S}\left(\mu_{R}^{2}\right), \mu_{R}^{2}, \mu_{F}^{2}, Q^{2}\right]=1+\alpha_{S}\left(\mu_{R}^{2}\right) Q\left(\mu_{F}^{2}, Q^{2}\right)
$$

is a hard factor, including the constant terms which are present in the NLO coefficient function but are not resummed in $\Delta_{N}^{(C)}$,

$$
d_{N}^{(C)}\left[\alpha_{S}\left(\mu_{R}^{2}\right), \mu_{R}^{2}, \mu_{F}^{2}, Q^{2}\right]=\alpha_{S}\left(\mu_{R}^{2}\right) Y\left(\mu_{F}^{2}, Q^{2}\right)
$$

is a remainder function, collecting the left-over NLO terms, suppressed at large $N$. The explicit expression for functions $Q\left(\mu_{F}^{2}, Q^{2}\right)$ and $Y\left(\mu_{F}^{2}, Q^{2}\right)$ can be read from the formulas in [4]. A similar expression holds for the resummed initial condition matched to the NLO result (see eq. (5.17) in ref. [4]).

According to the $\ln R$-matching, functions $K^{(C)}$ and $d^{(C)}$ are to be replaced by exponential functions of their $\mathcal{O}\left(\alpha_{S}\right)$ terms and eq.(2.13) should read:

$$
\begin{aligned}
C_{N}^{\mathrm{res}}\left[\alpha_{S}\left(\mu_{R}^{2}\right), \mu_{R}^{2}, \mu_{F}^{2}, Q^{2}\right] & =\exp \left[\alpha_{S}\left(\mu_{R}^{2}\right) Q\left(\mu_{F}^{2}, Q^{2}\right)\right] \times \Delta_{N}^{(C)}\left[\alpha_{S}\left(\mu_{R}^{2}\right), \mu_{R}^{2}, \mu_{F}^{2}, Q^{2}\right] \\
& \times \exp \left[\alpha_{S}\left(\mu_{R}^{2}\right) Y\left(\mu_{F}^{2}, Q^{2}\right)\right]
\end{aligned}
$$

From eq. (2.16), one can easily check that the logarithms of NLO and resummed functions are actually matched. In particular, eq. (2.16) differs from (2.13) only by terms of $\mathcal{O}\left(\alpha_{S}^{2}\right)$ or higher, but it is smoother at small and large values of $N(x)$, thanks to the exponential functions in eq. (2.16). It was in fact pointed out in [4 that, since the remainder function contains terms $\sim \ln x$ and $\sim \ln (1-x)$, the physical differential cross sections exhibit oscillating behaviour near $x \simeq 0$ and $x \simeq 1$. Exponentiating the $\mathcal{O}\left(\alpha_{S}\right)$ contributions to the remainder function should therefore improve the prediction for small and large values of $x$. The $\ln R$-matching prescription will be adopted in the following even for the resummed initial condition of the perturbative fragmentation function.

The $c$-quark spectrum will finally read in $N$-space as follows:

$$
\begin{aligned}
\sigma_{N}^{c}\left[\alpha_{S}\left(\mu_{0 R}^{2}\right), \alpha_{S}\left(\mu_{R}^{2}\right), \mu_{0 R}^{2}, \mu_{R}^{2}, \mu_{0 F}^{2}, \mu_{F}^{2}, m_{c}^{2}, Q^{2}\right] & =C_{N}^{\mathrm{res}}\left[\alpha_{S}\left(\mu_{R}^{2}\right), \mu_{R}^{2}, \mu_{F}^{2}, Q^{2}\right] \\
& \times E_{N}\left[\alpha_{S}\left(\mu_{0 F}^{2}\right), \alpha_{S}\left(\mu_{F}^{2}\right)\right] \\
& \times D_{N}^{\text {ini,res }}\left[\alpha_{S}\left(\mu_{0 R}^{2}\right), \mu_{0 R}^{2}, \mu_{0 F}^{2}, m_{c}^{2}\right] .
\end{aligned}
$$

In eq. (2.17), $E_{N}\left[\alpha_{S}\left(\mu_{0 F}^{2}\right), \alpha_{S}\left(\mu_{F}^{2}\right)\right]$ is the DGLAP operator for an evolution between the scales $\mu_{0 F}$ and $\mu_{F}$. Throughout this paper, we shall implement $E_{N}\left[\alpha_{S}\left(\mu_{0 F}^{2}\right), \alpha_{S}\left(\mu_{F}^{2}\right)\right]$ in the non-singlet approximation; its explicit expression can be found, e.g., in ref. [7]. 


\section{Effective coupling constant}

We shall include non-perturbative corrections to charm fragmentation using, as in [4], a model, based on an extension of refs. [5, 6], which includes power corrections via an effective strong coupling constant, and does not introduce any further parameter to be tuned to experimental data. We review below the main points of our model.

As discussed in ref. [28], in resummed calculations the momentum-independent coupling constant is replaced by the following integral over the discontinuity of the gluon propagator:

$$
\alpha_{S} \rightarrow \frac{i}{2 \pi} \int_{0}^{k^{2}} d s \operatorname{Disc}_{s} \frac{\alpha_{S}(-s)}{s}
$$

where $k^{2}$ is the gluon transverse momentum relative to the emitter, defined, e.g., as in eq. (2.7). In eq. (3.1) the discontinuity is given by:

$$
\operatorname{Disc}_{s} F(s)=\lim _{\epsilon \rightarrow 0^{+}}[F(s+i \epsilon)-F(s-i \epsilon)] .
$$

At LO, e.g., $\alpha_{S}(-s)$ reads:

$$
\alpha_{S, \mathrm{LO}}(-s)=\frac{1}{\beta_{0}\left[\ln \left(|s| / \Lambda^{2}\right)-i \pi \Theta(s)\right]},
$$

where $\beta_{0}=\left(33-2 n_{f}\right) /(12 \pi)$ is the first-order term of the QCD $\beta$-function, $n_{f}$ is number of active flavours, and $\Lambda$ is the QCD scale, e.g., in the $\overline{\mathrm{MS}}$ renormalization scheme.

The integral (3.1) is usually carried out neglecting the imaginary part, $\sim i \pi$, in the denominator of $\alpha_{S}(-s)$, i.e. assuming

$$
\ln \frac{|s|}{\Lambda^{2}} \gg \pi
$$

in eq. (3.3). The approximation (3.4) allows one to avoid the Landau pole, so that the integral (3.1) turns out to be roughly equal to the strong coupling constant evaluated at the upper integration limit:

$$
\frac{i}{2 \pi} \int_{0}^{k^{2}} d s \operatorname{Disc}_{s} \frac{\alpha_{S}(-s)}{s} \simeq \alpha_{S}\left(k^{2}\right)
$$

In fact, resummed formulas typically use the transverse momentum $k^{2}$ as the scale of the strong coupling constant 18].

As in [4], we shall follow a different approach and avoid the Landau pole by using in eq. (3.1) a regularized coupling constant $\bar{\alpha}_{S}$, defined as follows [5, 33]:

$$
\bar{\alpha}_{S}\left(k^{2}\right)=\frac{1}{2 \pi i} \int_{0}^{\infty} \frac{d s}{s+k^{2}} \operatorname{Disc}_{s} \alpha_{S}(-s) .
$$

Inserting in (3.6) the LO expression (3.3) and performing the integration, we obtain:

$$
\bar{\alpha}_{S, \mathrm{LO}}\left(k^{2}\right)=\frac{1}{\beta_{0}}\left[\frac{1}{\ln \left(k^{2} / \Lambda^{2}\right)}-\frac{\Lambda^{2}}{k^{2}-\Lambda^{2}}\right] .
$$


If we compare eq. (3.7) with the LO standard coupling, i.e.

$$
\alpha_{S, \mathrm{LO}}\left(k^{2}\right)=\frac{1}{\beta_{0} \ln \left(k^{2} / \Lambda^{2}\right)},
$$

we learn that in eq. (3.7) a power-suppressed term, relevant at small $k^{2}$, has subtracted off the Landau pole $k^{2}=\Lambda^{2}$, which is instead present in (3.8). At large $k^{2}, \bar{\alpha}_{S}\left(k^{2}\right)$ is nonetheless still roughly equal to $\alpha_{S}\left(k^{2}\right)$. Such results can be generalized to higher accuracy levels, using the two- and three-loop beta function, as done in [ [-

The effective coupling constant $\tilde{\alpha}_{S}\left(k^{2}\right)$ will be still defined as in eq.(3.1), but using the analytic coupling (3.6) in the integrand function:

$$
\tilde{\alpha}_{S}\left(k^{2}\right)=\frac{i}{2 \pi} \int_{0}^{k^{2}} d s \operatorname{Disc}_{s} \frac{\bar{\alpha}_{S}(-s)}{s} .
$$

Using the LO result (3.7), we can perform the integral (3.9) and obtain our LO effective coupling constant:

$$
\tilde{\alpha}_{S, \mathrm{LO}}\left(k^{2}\right)=\frac{1}{\beta_{0}}\left\{\frac{1}{2}-\frac{1}{\pi} \arctan \left[\frac{\ln \left(k^{2} / \Lambda^{2}\right)}{\pi}\right]\right\} .
$$

The NLO and NNLO expressions of $\tilde{\alpha}_{S}\left(k^{2}\right)$ can be found in [. $\mid$ ]. It is straightforward to show that eq. (3.10), as well as its higher-order generalizations, is free from the Landau pole and includes power-suppressed contributions at small momenta. Also, as discussed in [26], eq. (3.9) accounts for absorptive effects due to gluon branching, since we are not neglecting any longer the imaginary part in the denominator of $\alpha_{S}(-s)$.

In principle, both analytic coupling constants (3.6) and (3.9) are possible candidates to model non-perturbative corrections ${ }^{2}$. However, as debated in 沟, it is only (3.9) which gives an acceptable description of $b$-fragmentation data and we shall therefore stick to $\tilde{\alpha}_{S}\left(k^{2}\right)$ to model power corrections to charm fragmentation as well.

The relation between effective and standard coupling constant for $\ln \left(k^{2} / \Lambda^{2}\right) \gg \pi$ reads:

$$
\tilde{\alpha}_{S}\left(k^{2}\right)=\alpha_{S}\left(k^{2}\right)-\frac{\left(\pi \beta_{0}\right)^{2}}{3} \alpha_{S}^{3}\left(k^{2}\right)+\mathcal{O}\left(\alpha_{S}^{4}\right) .
$$

From eq. (3.11) we learn that at high energy the difference between $\tilde{\alpha}_{S}\left(k^{2}\right)$ and $\alpha_{S}\left(k^{2}\right)$ starts from $\mathcal{O}\left(\alpha_{S}^{3}\right)$. Moreover, eq. (3.11) dictates that, when employing the effective coupling constant, we will have to redefine the soft-resummation coefficients from order $\alpha_{S}^{3}$ on. As anticipated in subsection 2.2, the NNLL coefficient $A^{(3)}$ of the $\mathcal{O}\left(\alpha_{S}^{3}\right)$ term of function $A\left(\alpha_{S}\right)$, entering in Eqs. (2.7) and (2.11), will get enhanced according to:

$$
A^{(3)} \rightarrow \tilde{A}^{(3)}=A^{(3)}+\frac{\left(\pi \beta_{0}\right)^{2}}{3} A^{(1)} .
$$

The other assumptions contained in our model are also detailed in ref. [4] and we do not report them here for the sake of brevity. We just point out that, when dealing with higher

\footnotetext{
${ }^{2}$ In the literature [4, 5$]$, one usually refers to $\bar{\alpha}_{S}\left(k^{2}\right)$ and $\tilde{\alpha}_{S}\left(k^{2}\right)$ as effective space- and time-like coupling constants, respectively.
} 
orders of $\tilde{\alpha}_{S}\left(k^{2}\right)$, we shall adopt the so-called 'power-expansion' choice, which implies that we shall evaluate the powers $\tilde{\alpha}_{S}^{n}\left(k^{2}\right)$ after computing the integral over the discontinuity:

$$
\tilde{\alpha}_{S}^{n}\left(k^{2}\right)=\left[\frac{i}{2 \pi} \int_{0}^{k^{2}} d s \operatorname{Disc}_{s} \frac{\bar{\alpha}_{S}(-s)}{s}\right]^{n} .
$$

On the contrary, the original proposal in [5] consisted in calculating the discontinuity of $\bar{\alpha}_{S}^{n}(-s)$ before integrating over $s$ ('non power-expansion' choice). As discussed in [4], the non-power expansion prescription would yield a rather poor description of $b$-fragmentation data.

The purpose of the present paper is indeed to push the effective-coupling model to lower energies and compare its predictions with data on $c$-flavoured hadron production. For a consistent comparison with the results obtained in the framework of $B$-hadron pro-

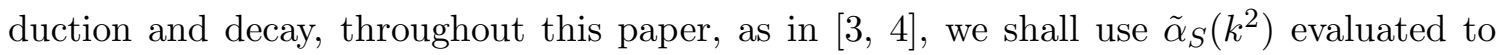
three-loop accuracy, everywhere in our calculation, i.e. in both coefficient function and perturbative fragmentation function. Hereafter, the effective coupling constant (3.9) will be our only source of non-perturbative corrections and we shall not introduce any further non-perturbative fragmentation function.

It was pointed out in [4 that power-correction effects in the initial condition of the perturbative fragmentation function are more relevant than in the coefficient function. The typical $c$-fragmentation scales at which the coupling constant is evaluated are, in fact, $C=Q \sqrt{1-x}$ in the coefficient function and $S=m_{c}(1-x)$ in the initial condition. $C$ and $S$ are the integration limits in the resummed exponents as well as the arguments of $\alpha_{S}$ in functions $B\left[\alpha_{S}\left(C^{2}\right)\right]$ and $D\left[\alpha_{S}\left(S^{2}\right)\right]$, appearing in the large- $x$ resummation expressions (2.7) and (2.11). If we calculate $C$ and $S$ for $Q=m_{Z}$ and $x=0.5$, where, as will be shown in the next section, the $D$-meson spectrum in $e^{+} e^{-}$annihilation is roughly peaked, we shall get $C \simeq 46 \mathrm{GeV}, S \simeq 0.9 \mathrm{GeV}, \tilde{\alpha}_{S}\left(C^{2}\right) \simeq 0.13$ and $\tilde{\alpha}_{S}\left(S^{2}\right) \simeq 0.35$. Therefore, non-perturbative corrections are more important in the initial condition, depending on $S$, than in the coefficient function. Comparing now the values of $\tilde{\alpha}_{S}$ at the charm- and bottom-mass scales, we find that $\tilde{\alpha}_{S}\left(m_{c}^{2}\right) \simeq 0.3$ is appreciably higher than $\tilde{\alpha}_{S}\left(m_{b}^{2}\right) \simeq 0.2$. However, it is interesting to notice that the scales $S$ and $C$, and hence $\tilde{\alpha}_{S}\left(S^{2}\right)$ and $\tilde{\alpha}_{S}\left(C^{2}\right)$, are roughly the same for bottom and charm production if evaluated at the maxima of the respective spectra at LEP, i.e. $x=0.5$ for $D$ - and $x=0.8$ for $B$-hadron energy distributions.

Before closing this section, we would like to stress that, in its current formulation, our parameter-free model works in the same fashion for $B$ as well $D$ mesons, up to the replacement $m_{b} \rightarrow m_{c}$. Also, our model does not distinguish among baryons and mesons, spin-1 and spin-0, charged and neutral hadrons. It was therefore argued in [4] that possible extensions of our model may consist in including a correcting term, so that

$$
\tilde{\alpha}_{S}\left(k^{2}\right) \rightarrow \tilde{\alpha}_{S}\left(k^{2}\right)+\delta \tilde{\alpha}_{S}\left(k^{2}\right),
$$

where $\tilde{\alpha}_{S}\left(k^{2}\right)$ is still the effective coupling discussed above, and $\delta \tilde{\alpha}_{S}\left(k^{2}\right)$ may depend, e.g., on whether we have baryons or mesons, B's or $D$ 's, and so on. The analysis which we shall undertake herafter should therefore be helpful to establish, for the time being, whether the contribution $\delta \tilde{\alpha}_{S}\left(k^{2}\right)$ is mandatory or not. 


\section{Results in $x$-space}

In this section we compare our results in $x$-space with experimental data on $c$-flavoured hadron production in $e^{+} e^{-}$annihilation. Hadronization effects will be accounted for by employing the analytic coupling constant (3.9) at NNLO. Whenever we use $\tilde{\alpha}_{S}\left(k^{2}\right)$ instead of the standard $\alpha_{S}\left(k^{2}\right)$, the charm-quark energy fraction will be replaced by its hadron-level counterpart:

$$
x_{D}=\frac{2 p_{D} \cdot Q}{Q^{2}},
$$

with $p_{D}$ being the momentum of a $D$-hadron. The $D$ spectrum in moment space will be written in a form analogous to eq.(2.17), up to $\alpha_{S} \rightarrow \tilde{\alpha}_{S}$ :

$$
\begin{aligned}
\sigma_{N}^{(D)}\left(\mu_{R}^{2}, \mu_{0 R}^{2}, \mu_{0 F}^{2}, \mu_{F}^{2}, m_{c}^{2}, Q^{2}\right) & =C_{N}^{\text {res }}\left[\tilde{\alpha}_{S}\left(\mu_{R}^{2}\right), \mu_{R}^{2}, \mu_{F}^{2}, Q^{2}\right] \times E_{N}\left[\tilde{\alpha}_{S}\left(\mu_{0 F}^{2}\right), \tilde{\alpha}_{S}\left(\mu_{F}^{2}\right)\right] \\
& \times D_{N}^{\text {ini,res }}\left[\tilde{\alpha}_{S}\left(\mu_{0 R}^{2}\right), \mu_{0 R}^{2}, \mu_{0 F}^{2}, m_{c}^{2}\right] .
\end{aligned}
$$

The $x$-space result is then recovered by performing an inverse Mellin transform:

$$
\sigma^{(D)}\left(x_{D} ; \mu_{R}^{2}, \mu_{0 R}^{2}, \mu_{0 F}^{2}, \mu_{F}^{2}, m_{c}^{2}, Q^{2}\right)=\int_{\gamma-i \infty}^{\gamma+i \infty} \frac{d N}{2 \pi i} x_{D}^{-N} \sigma_{N}^{(D)}\left(\mu_{R}^{2}, \mu_{0 R}^{2}, \mu_{0 F}^{2}, \mu_{F}^{2}, m_{c}^{2}, Q^{2}\right),
$$

where $\gamma$ is a positive constant. As discussed in 四, since the effective $\tilde{\alpha}_{S}\left(k^{2}\right)$ does not exhibit the Landau pole any longer, we do not need any prescription, such as the wellknown minimal prescription [29], to avoid the Landau pole in the integration (4.3). The integral will be performed in a numerical way, along the lines of [4]; it was checked that the results are stable when varying the integration contour, i.e. the constant $\gamma$.

As in ref. [30], we shall consider LEP data from the ALEPH collaboration [31], taken at the $Z^{0}$ pole, and data from the CLEO [32] and BELLE [33] experiments, at the $\Upsilon(4 S)$ resonance. We shall investigate neutral as well as charged $D$ and $D^{*}$ mesons; in fact, we just pointed out that our model does not distinguish the hadron electric charge or spin.

As discussed in [30], electromagnetic initial-state radiation (ISR) effects can modify the shape of charmed-meson spectra. Such effects are important especially at $B$-factories, where the emission of photons from the $e^{+} e^{-}$pair, whose rate is $\sim \alpha \ln \left(Q^{2} / m_{e}^{2}\right), m_{e}$ being the electron mass, may significantly decrease the energy in the centre-of-mass system. The CLEO and BELLE data did not account for such effects, which were instead implemented in the analysis [30]. In the following, we shall compare with data corrected for ISR effects: a discussion on the impact of such contributions on $D$-spectra in $x$ - and $N$-spaces can be found in [30]. Such effects were also implemented to correct the ALEPH data, but it was understood that at the $Z^{0}$ pole they are quite negligible.

The non-perturbative model based on the effective coupling constant (3.9) does not have any free parameter to be tuned to the data which we shall consider. We shall nonetheless vary the parameters entering in the perturbative calculation in such a way to give an estimate of the theoretical uncertainty on our prediction. We change each quantity separately, keeping the others to their default values, in such a way to avoid too many runs.

Following [⿶], the default values of our perturbative parameters will be $\mu_{R}=\mu_{F}=Q$ and $\mu_{0 R}=\mu_{0 F}=m_{c}$, where $\mu_{R}$ and $\mu_{F}$ are the renormalization and factorization scales 
in the coefficient function, and $\mu_{0 R}$ and $\mu_{0 F}$ in the initial condition of the perturbative fragmentation function. The hard scale will be $Q=m_{Z}$ or $m_{\Upsilon(4 S)}$ at LEP or $B$-factories, with $m_{Z}=91.19 \mathrm{GeV}$ and $m_{\Upsilon(4 S)}=10.58 \mathrm{GeV}$. We shall vary $\mu_{R}$ and $\mu_{F}$ between $Q / 2$ and $2 Q, \mu_{0 R}$ and $\mu_{0 F}$ between $m_{c} / 2$ and $2 m_{c}$. As in 俰, we shall let $\alpha_{S}\left(m_{Z}^{2}\right)$ run in the range $0.117<\alpha_{S}\left(m_{Z}^{2}\right)<0.121$, using $\alpha_{S}\left(m_{Z}^{2}\right)=0.119$ as our default value. The corresponding variation range of the effective coupling constant is $0.115<\tilde{\alpha}_{S}\left(m_{Z}^{2}\right)<0.119$. For the purpose of $m_{c}$, as thoroughly discussed in [4], using the pole or the $\overline{\mathrm{MS}}$ heavy-quark mass definition in the initial condition is equivalent for calculations relying on the NLO/NLL approximation. However, in the NNLL large- $x$ resummation of the initial condition, and in particular in the definition of the coefficient $D^{(2)}$ in eq. (2.11), we are employing results of the NNLO computation in [14], which uses the heavy-quark pole mass. Hence, we should use the charm pole mass as well. Nonetheless, as pointed out in 伆, when we use the effective coupling constant to describe hadronization corrections, it is not uniquely determined whether $m_{c}$ should be the quark or the hadron mass. As done for the purpose of the bottom-quark mass, we shall adopt a conservative choice and vary $m_{c}$ in the range $1.5 \mathrm{GeV}<m_{c}<2.1 \mathrm{GeV}$, that includes the current estimations for the charm pole mass as well as $D$-hadron masses [34]. Our default value will be $m_{c}=1.8 \mathrm{GeV}$.

As for the DGLAP evolution operator, when evolving from $\mu_{0 F} \simeq m_{c}$ to $\mu_{F} \simeq Q$, one typically crosses the bottom-quark mass threshold $m_{b}$. ref. [35] computed at NLO the matching conditions for the perturbative fragmentation function when crossing heavyflavour thresholds. In our study, however, since we are working in the non-singlet approximation and we are not accounting for gluon splitting and flavour mixing, we shall neglect such matching conditions. In fact, we checked that our results change very little according to whether we set in the DGLAP evolution operator, e.g, $n_{f}=4$ or $n_{f}=5$ as the number of active flavours. In any case, in our phenomenological analysis, whenever we have $\mu_{0 F}<m_{b}<\mu_{F}$, we shall implement the following factorized form for the non-singlet DGLAP evolution operator:

$$
E_{N}\left[\tilde{\alpha}_{S}\left(\mu_{0 F}^{2}\right), \tilde{\alpha}_{S}\left(\mu_{F}^{2}\right)\right]=E_{N}\left[\tilde{\alpha}_{S}\left(\mu_{0 F}^{2}\right), \tilde{\alpha}_{S}\left(m_{b}^{2}\right)\right] \times E_{N}\left[\tilde{\alpha}_{S}\left(m_{b}^{2}\right), \tilde{\alpha}_{S}\left(\mu_{F}^{2}\right)\right],
$$

with $n_{f}=4$ and $n_{f}=5$ below and above the bottom-quark mass threshold, respectively. The $b$-quark mass will be varied in the range $4.7 \mathrm{GeV}<m_{b}<5.3 \mathrm{GeV}$, as in [4], with $m_{b}=5 \mathrm{GeV}$ being our default value. Elsewhere in our calculation, $n_{f}$ will be consistently chosen according to the energy scale we are dealing with.

\subsection{Comparison with ALEPH data}

We shall first consider ALEPH data on $D^{*+}$ production. As detailed in [31], such mesons can be in general produced from a $Z^{0} \rightarrow c \bar{c}$ decay, from the decay of a primary $b$-flavoured hadron produced in $Z^{0} \rightarrow b \bar{b}$, from gluon splitting to $c \bar{c}$ or $b \bar{b}$ pairs, which subsequently hadronize or decay into a $D^{*+}$. The ALEPH Collaboration was able to subtract the $Z^{0} \rightarrow b \bar{b}$ and gluon-splitting contributions off and published the spectrum of $D^{*+}$ mesons coming only from the $c \bar{c}$ primary source. In the following, we shall compare the predictions of our model with such a subsample, which will allow us to neglect secondary charm 
production in the perturbative calculation as well as the singlet component of the DGLAP evolution operator, which in principle should play a role at LEP energies. Indeed, it was found out in [30] that implementing the singlet contribution does have an effect at $x_{D}<0.4$, and actually worsens the comparison with the ALEPH $D^{*+}$ data coming from direct $c \bar{c}$ production (see figures 13 and 14 in ref. [30]).

In figure 1 we present the spectrum given by our model, along with the $D^{*+}$ ALEPH data, and investigate the dependence on the factorization scales $\mu_{F}$ and $\mu_{0 F}$ (figure 1 1 (a)), and on the choice of $\alpha_{S}\left(m_{Z}^{2}\right)$ and $m_{c}$ (figure 1 (b)). For the sake of comparison, both data and theoretical predictions are normalized to unity. As already observed in [4], the dependence on $\mu_{0 F}$, the scale entering in the initial condition of the perturbative fragmentation function, is fairly large, while the impact of the choice of $\mu_{F}$ is pretty small. In particular, setting a lower value of $\mu_{0 F}$, e.g. $\mu_{0 F}=m_{c} / 2$, tends to deplete the small- $x_{D}$ region of the spectrum and to enhance the event fraction around the peak. Also, the peak is slightly shifted to higher $x_{D}$ if we choose $\mu_{0 F}=m_{c} / 2$. The prediction obtained for $\mu_{0 F}=2 m_{c}$ reproduces quite well the low- $x_{D}$ data, while discrepancies are still present in the middle-high range.

The dependence on $\alpha_{S}\left(m_{Z}^{2}\right)$ and $m_{c}$ is also quite relevant, as can be learned from figure 1 (b). In particular, a low value of $m_{c}$, i.e. $m_{c}=1.5 \mathrm{GeV}$, consistent with the quark mass rather than the $D^{*+}$-meson mass, gives a pretty good description of the peak, but it worsens the comparison for $x_{D}>0.7$. On the contrary, a high value of $m_{c}$, such as 2.1 $\mathrm{GeV}$, significantly moves the peak towards large $x_{D}$ and worsens the overall comparison. As for the effect of the variation of $\alpha_{S}\left(m_{Z}^{2}\right)$, we find that it shifts the position of the peak: the lower $\alpha_{S}\left(m_{Z}^{2}\right)$, the higher the value of $x_{D}$ at which the $D$ spectrum is peaked. The dependence on the renormalization scales $\mu_{R}$ and $\mu_{0 R}$ is very little, and we do not present the corresponding plots for the sake of brevity. We also varied $m_{b}$, the bottom-quark mass entering in eq. (4.4), but found out that it has negligible impact on the energy distribution.

Overall, we can say that our model gives an acceptable description of the raise at low and average values of $x_{D}$, while discrepancies are present around the peak, unless one sets a relatively low value for $m_{c}$, and at very large $x_{D}$. Our curves tend to be harder than the data and approach zero at large $x_{D}$ more rapidly. Although the comparison at very large $x_{D}$ is not completely satisfactory, using the $\ln R$-matching prescription, discussed in subsection 2.2., has nonetheless improved the spectrum near the endpoint $x_{D}=1$, as it is smoother and not oscillating any longer. We checked that if we had used the standard matching between NLO and resummed expressions as in [4], the charmed-meson distributions would have become negative for $x_{D} \gtrsim 0.9$.

In any case, we are aware that our model, based on an extrapolation of perturbation theory, up to the replacement of the coupling constant $\alpha_{S}\left(k^{2}\right) \rightarrow \tilde{\alpha}_{S}\left(k^{2}\right)$, cannot be completely reliable at very large $x_{D}$. One can roughly estimate [11] $x_{D, \max } \simeq 1-\Lambda / m_{c} \simeq 0.85$ the maximum value of $x_{D}$ at which our model, or any model based on simple parametrizations of power corrections, such as the non-perturbative fragmentation functions [1, 2], can be trusted. In fact, the authors of ref. [30] managed to improve the comparison at large $x_{D}$, but they had to introduce a further free parameter which they tuned to data. In detail, 

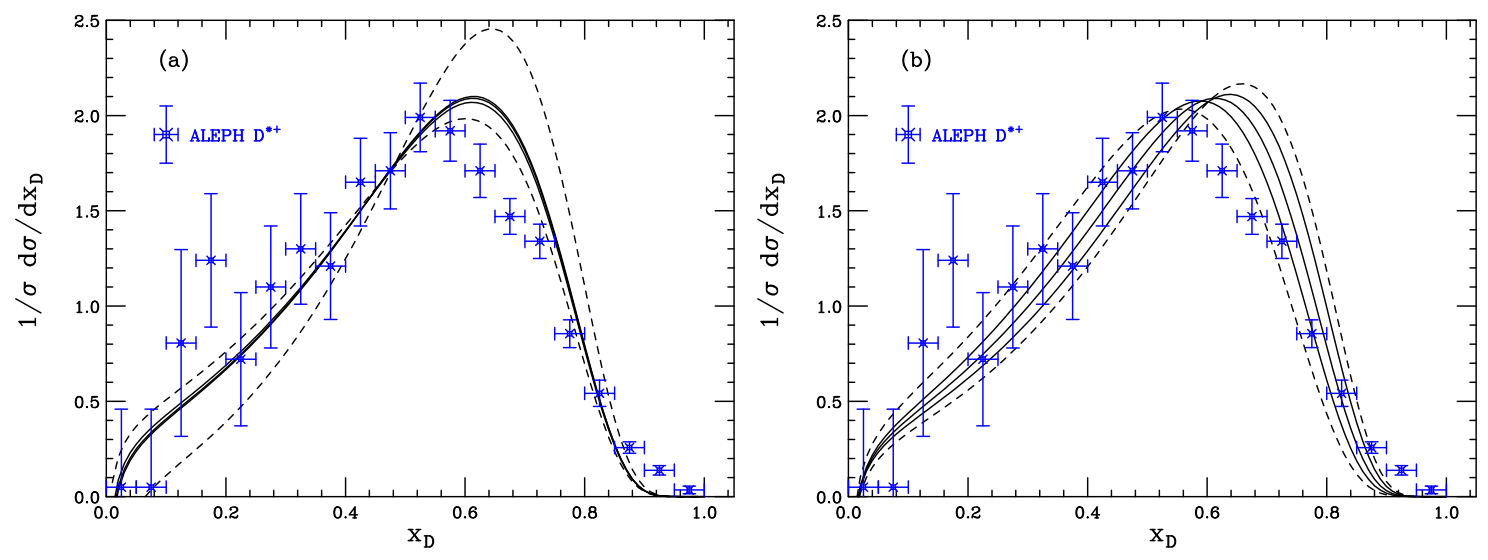

Figure 1: Comparison of the prediction yielded by our model with data from ALEPH on $D^{*+}$ production a LEP. In (a) we investigate the dependence on $\mu_{F}$ (solid lines) and $\mu_{0 F}$ (dashes); in (b) the effect of the choice of $\alpha_{S}\left(m_{Z}^{2}\right)$ (solid) and $m_{c}$ (dashes). Such quantities are varied as discussed in the text.

they replaced the Mellin variable $N$ according to:

$$
N \rightarrow N \frac{1+f / N^{\prime}}{1+f N / N^{\prime}}
$$

where $N^{\prime}=\exp \left[1 /\left(\beta_{0} \alpha_{S}\left(\mu_{R}^{2}\right)\right)\right]$ in the coefficient function and $N^{\prime}=\exp \left[1 /\left(2 \beta_{0} \alpha_{S}\left(\mu_{0 R}^{2}\right)\right)\right]$ in the initial condition. ref. [30] used then $f=1.25$ in its phenomenological analysis, as this choice led to good fits to charm-fragmentation data. In principle, we could also perform the replacement (4.5) and tune $f$. In fact, modifying the energy distribution at large $x_{D}$ will also have an impact at smaller values of $x_{D}$, since we have kept the normalization of data and theory curves fixed to unity. However, in this way our model would lose its crucial feature to be free from tunable parameters. Furthermore, given the theoretical uncertainty on our prediction, the value of $f$ will depend on the particular set of perturbative parameters chosen. Therefore, for the time being, we prefer to stick to our parameter-free modelling of the hadronization and to postpone a more careful investigation of the very large- $x_{D}$ regime of our spectra to future work, with the NNLO corrections implemented. In any case, we should never forget that, for the sake of consistency, whenever we modify the perturbative accuracy or the non-perturbative model, we should always reconsider the studies on $B$-hadron production and decay and check whether the results obtained in refs. [3] and [4] still hold.

In the present analysis, as in ref. 朋, we discard few points at very large $x_{D}$ and limit ourselves to $x_{D} \leq 0.85$ when evaluating the $\chi^{2}$ from the comparison with the data. Even in this range, using our default values for the parameters in the parton-level computation, we are not able to acceptably reproduce the data, as we obtain $\chi^{2} /$ dof $=56.47 / 17$. A better description of the data is nonetheless obtained if, e.g., we keep all quantities to their default values, but set $\mu_{0 F}=2 m_{c}\left(\chi^{2} /\right.$ dof $\left.=27.18 / 17\right)$ or $\alpha_{S}\left(m_{Z}^{2}\right)=0.121\left(\chi^{2} /\right.$ dof $=$ $30.52 / 17)$. Setting $m_{c}=1.5 \mathrm{GeV}$, we find $\chi^{2} /$ dof $=32.29 / 17$. As we are not fitting any 
non-perturbative parameter to the data, such values of $\chi^{2}$ are acceptable. Also, they are of similar magnitude to those obtained in 4 from the comparison with $B$-hadron energy distributions at the $Z^{0}$ pole for $x_{B}<1-\Lambda / m_{b}$.

The overall impact of the inclusion of non-perturbative corrections at LEP energies via our model can be learned from figure 2, where we present our most significant predictions, i.e. the ones obtained with $\mu_{0 F}=2 m_{c}$ and $m_{c}=1.8 \mathrm{GeV}$ (solid line), and with $\mu_{0 F}=$ $m_{c}$ and $m_{c}=1.5 \mathrm{GeV}$ (dotted), keeping the other quantities to their default values. In figure 2 we also show the ALEPH $D^{*+}$ data and the purely perturbative results of ref. [11], where the authors used the standard coupling constant and resummed NLL soft and collinear contributions to both coefficient function and perturbative fragmentation function. The role played by power corrections is clearly remarkable throughout all $x_{D^{-}}$ spectrum, and is essential to obtain an acceptable description of the data. In fact, the parton-level calculation of [1], which is the same as the one employed in [30, needs to be convoluted with a non-perturbative fragmentation function to reproduce the data. We can also note in figure 2 that, while setting $\mu_{0 F}=2 m_{c}$ and $m_{c}=1.8 \mathrm{GeV}$ gives the lowest $\chi^{2}$, the data around the peak are better described if we instead choose $m_{c}=1.5 \mathrm{GeV}$ and $\mu_{0 F}=m_{c}$.

Before closing this subsection, we remind that the possible reasons determining the fairly large theoretical uncertainties were already listed and detailed in 耘. In particular, we have resummed large- $x$ contributions to the coefficient function and initial condition in the NNLL approximation, but we have still matched the resummation to the NLO exact results, thus generating a mismatch between the NNLL terms in the resummed exponents $\left(\sim \alpha_{S}^{n} \ln N\right.$, etc.) and the remainder functions. We believe that the uncertainties should be milder if we used the exact NNLO results [12, 13, 14, 16]. Moreover, lower theoretical errors should be expected if we also employed NNLL non-singlet DGLAP evolution equations, using NNLO non-singlet splitting functions 15 .

With respect to the analysis on $B$-hadron production, the effect of the choice of scales and masses is here even more relevant: the dependence on such quantities is typically logarithmic, hence larger once they vary around $m_{c}$ rather than $m_{b}$. It is however interesting to notice that, unlike the comparison with the $B$-hadron data, where setting $\mu_{0 F}=m_{b} / 2$ gave the best description of the data [4], the charm-fragmentation data seem to prefer a quite high value of $\mu_{0 F}$, since $\mu_{0 F}=2 m_{c}$ yields the lowest $\chi^{2}$. We believe that a full NNLO/NNLL analysis should clarify this issue as well.

\subsection{Comparison with CLEO and BELLE data}

We would like to compare the predictions of our model with the data on $D^{0}, D^{* 0}$ and $D^{*+}$ production from the experiments CLEO [32] and BELLE [33], collected at the $\Upsilon(4 S)$ resonance. In fact, since the value of the hard scale is much smaller than at LEP, such a comparison will help to shed light on the performance of our model and calculation at lower energies. Furthermore, ref. [30], using a NLO/NLL calculation and a non-perturbative fragmentation function with three parameters, managed to fit all $B$-factory data, whereas some discrepancies were found with respect to the ALEPH data after evolving to LEP energies. Our case is clearly different, as our non-perturbative model is not tunable to 


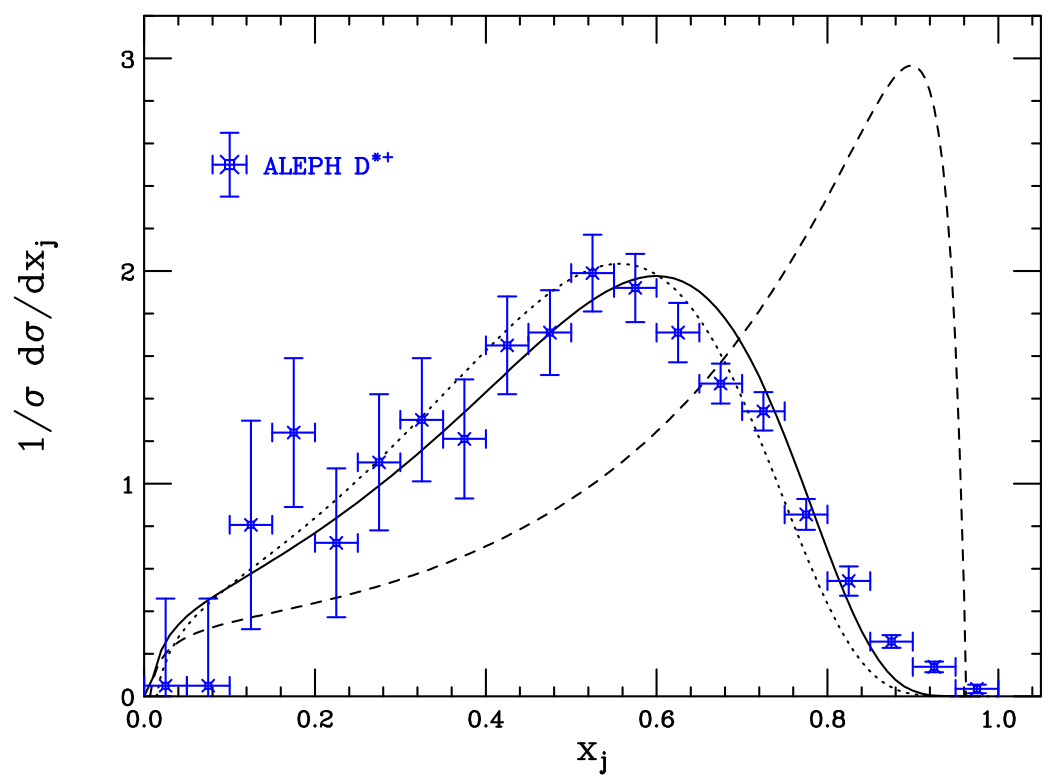

Figure 2: Results on charmed-hadron production at LEP $(j=D)$, setting $\mu_{0 F}=2 m_{c}$ and $m_{c}=1.8 \mathrm{GeV}$ (solid), $m_{c}=1.5 \mathrm{GeV}$ and $\mu_{0 F}=m_{c}$ (dots), compared with the perturbative parton-level calculation of [11] ( $j=c$, dashes) and the ALEPH $D^{*+}$ data.

data, but it will be nonetheless cumbersome to investigate how our predictions fare with respect to the different data sets at the $\Upsilon(4 S)$ resonance and estimate the theoretical uncertainty.

In figure 3 we present the comparison with CLEO and BELLE data on $D^{0}$ production, corrected for ISR effects. As pointed out in [30], at the $\Upsilon(4 S)$ resonance the contribution of $c \bar{c}$ pair production via gluon splitting is negligible, hence it is safe sticking to the nonsinglet approximation of the DGLAP evolution equations, as done for analysis at LEP energies. The data sets which we consider are separately normalized to 1 , for the sake of a consistent comparison with the theory curves, whose first moment reads, by definition, $\sigma_{N=1}=1$. We vary renormalization and factorization scales, $m_{c}$ and $\alpha_{S}\left(m_{Z}^{2}\right)$ along the lines of our comparison with ALEPH. figure 3 (a) exhibits the dependence on $\mu_{F}$ and $\mu_{0 F}$; figure 3 (b) the one on $\alpha_{S}\left(m_{Z}^{2}\right)$ and $m_{c}$. We do not present the effect of changing $\mu_{R}, \mu_{0 R}$ and $m_{b}$, since it is very little, as already found at the $Z^{0}$ pole.

Unlike the comparison with the ALEPH data, where, though within the experimental and theoretical uncertainties, we succeeded in getting a reasonable fit of the data, our prediction lies quite far from the CLEO and BELLE $D^{0}$ data and there is no choice of parameters and scales, within our ranges, which can accommodate the experimental data. In fact, such data exhibit very small errors and, even if we limit our analysis to $x_{D}<0.85$, as we did before, we still obtain quite large $\chi^{2}$, typically $\chi^{2} /$ dof $\gtrsim \mathcal{O}(10)$. It is nonetheless interesting to notice that the best comparison is obtained for $m_{c}=1.5 \mathrm{GeV}$ : in this case, one is able at least to reproduce the rise of the spectrum up to $x_{D} \simeq 0.6$, but still uncapable of describing the peak and the large- $x_{D}$ tail. As pointed out when comparing with ALEPH, a full NNLO/NNLL analysis is mandatory to reduce the theoretical error and should shed 
light on the dependence on the quark (meson) mass as well. Ref. [30] also presented $D^{+}$ data from CLEO and BELLE; the comparison with our predictions is however qualitatively similar to the one presented in figure 3 and we do not show it for brevity.
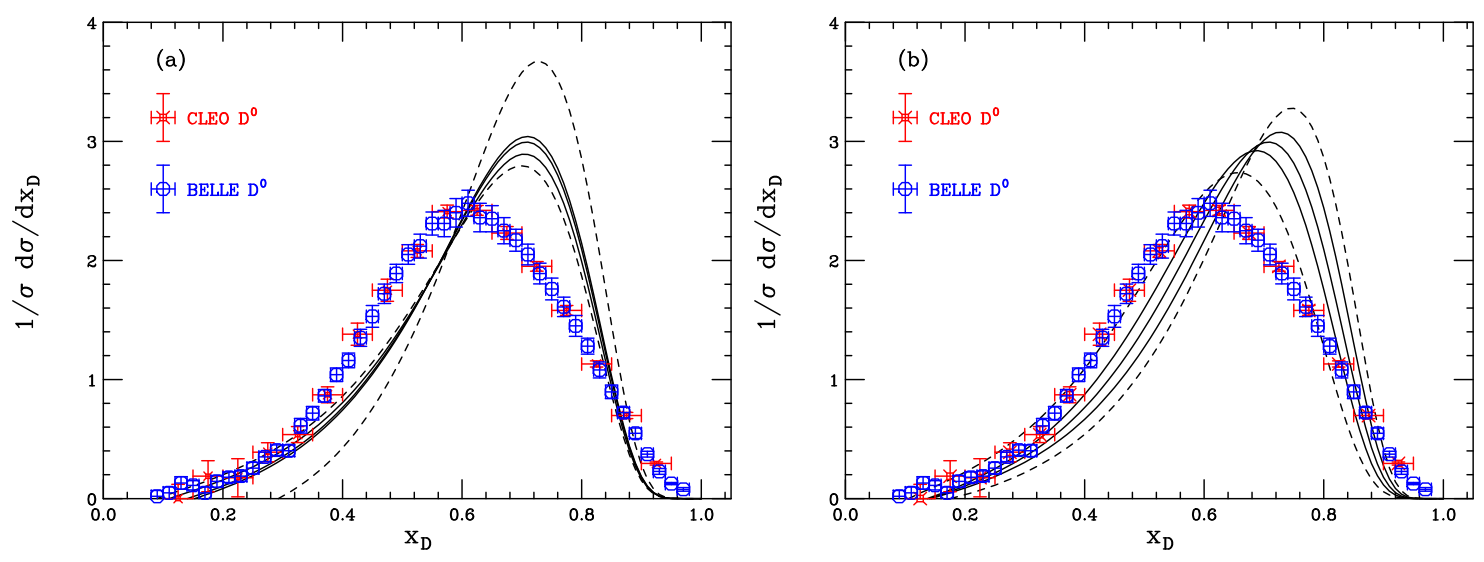

Figure 3: As in figure 1, but comparing with $D^{0}$ data from CLEO and BELLE experiments. In (a) we vary the factorization scales $\mu_{F}$ (solid) and $\mu_{0 F}$ (dashes); in (b) $\alpha_{S}\left(m_{Z}^{2}\right)$ (solid) and $m_{c}$ (dashes).

We present in figure 4 the comparison of the predictions yielded by our model with CLEO and BELLE data on $D^{* 0}$-meson production. The comparison with the CLEO $D^{* 0}$ data, which are affected by pretty small errors, is quite unsatisfactory and the $\chi^{2}$ values high. The BELLE spectrum instead exhibits larger errors, so that we are able to compare with the data at $x_{D}<0.85$ with quite small $\chi^{2}$ values. With our default parametrization, we obtain $\chi^{2} /$ dof $=45.23 / 36$, while an even lower result, $\chi^{2} /$ dof $=32.10 / 36$, is obtained if we set $\mu_{0 F}=2 m_{c}$, the same choice leading to the best fit to the ALEPH $D^{*+}$ spectrum.

We finally show in figure 5 the comparison of our predictions with the data on $D^{*+}$ production at CLEO and BELLE. Following [30], as far as the BELLE data are concerned, we present separately the spectra of the mesons decaying according to $D^{*+} \rightarrow D^{+}$and $D^{*+} \rightarrow D^{0}$, with the former presenting larger errors. The comparison is qualitatively similar to figure 4 , with our model capable of describing well the data up to $x_{D} \simeq 0.6$, but failing to reproduce the peak and the large- $x_{D}$ tail. Drawing a parallel between figure 5 and figure 1, where our model, though within the uncertainties, led to a better comparison with respect to the ALEPH $D^{*+}$ data, one may argue that some major problems with our approach seem to appear once the process hard scale decreases. Later on, in section 6 , we shall comment more about possible extensions of our calculation and non-perturbative model, which may eventually improve the comparison with the $B$-factory data in $x$-space.

As done when comparing with ALEPH, we show in figure 6 the prediction leading to the best fit to the $B$-factory data, i.e. the one obtained for $\mu_{0 F}=2 m_{c}$, along with the BELLE $D^{* 0}$ spectrum and the NLO/NLL perturbative prediction from ref. [11]. We note that the parton-level result is sharply peaked at large $x$, even more than in figure 2: 

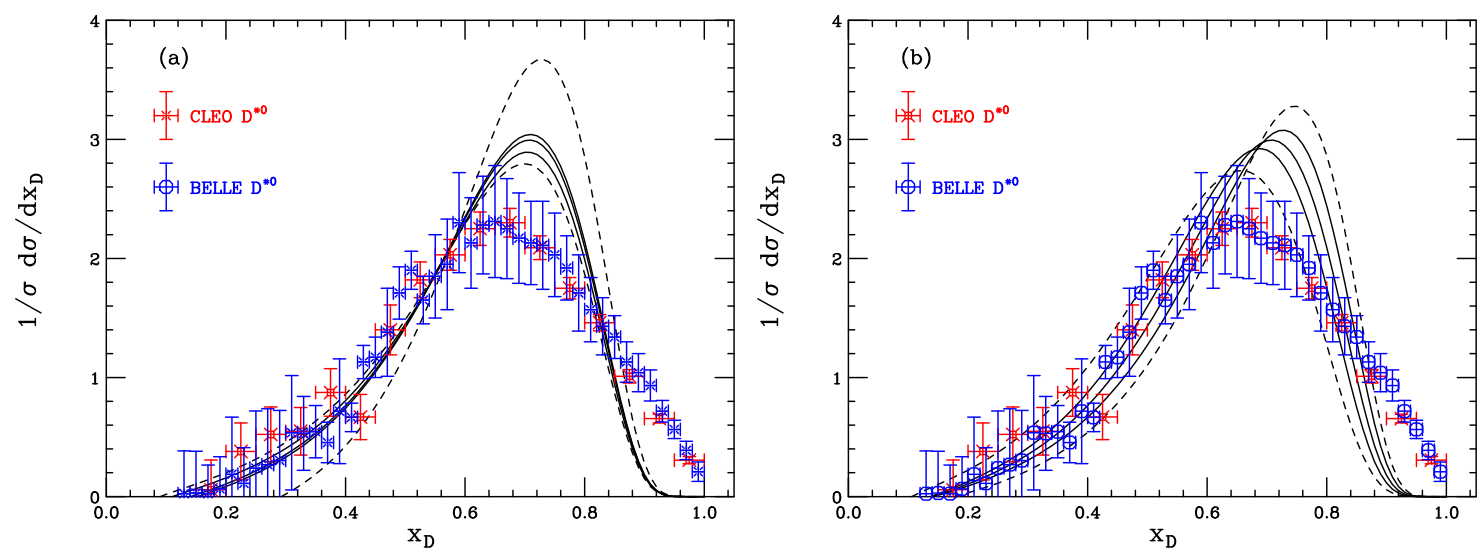

Figure 4: Comparison of the prediction yielded by our effective-coupling model with $D^{* 0}$ data from BELLE and CLEO. The scales are varied as in figures 1 and 3 .
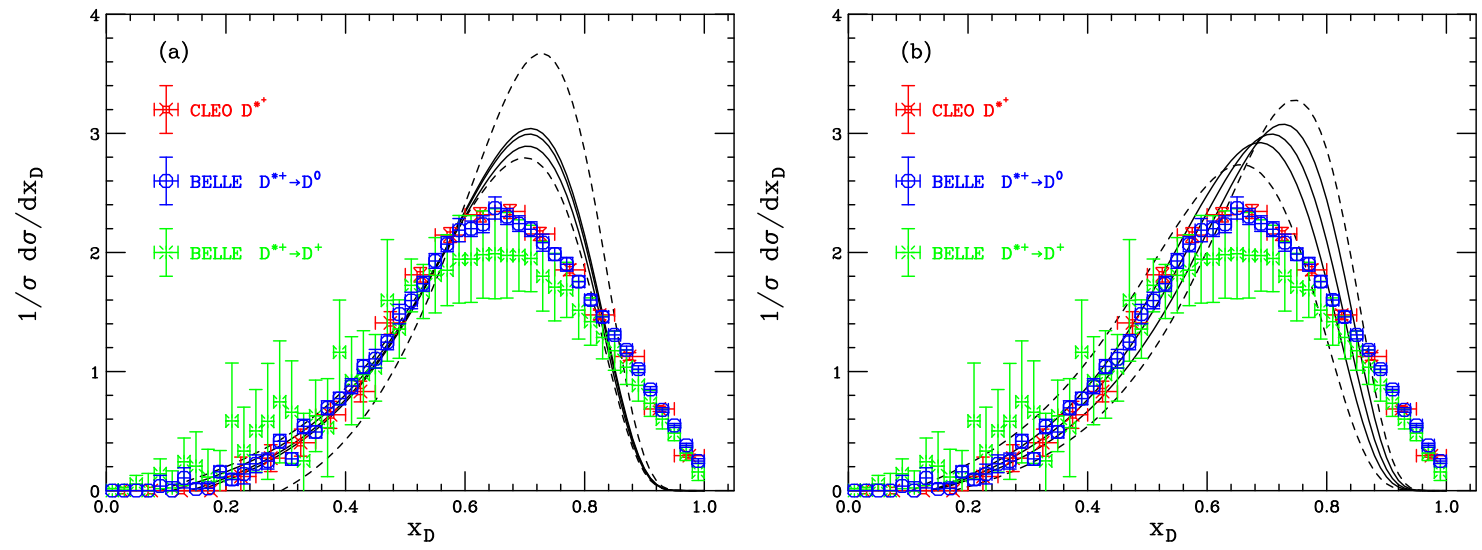

Figure 5: As in figures 3 4 , but comparing with the CLEO and BELLE data on $D^{*+}$ production. When presenting the BELLE data, we distinguish the $D^{*+} \rightarrow D^{0}$ from the $D^{*+} \rightarrow D^{0}$ decay mode.

in fact, the smaller phase space available at the $\Upsilon(4 S)$ resonance with respect to the $Z^{0}$ pole enhances the probability of producing $c \bar{c}$ pairs near the threshold $x=1$. Overall, the impact of non-perturbative corrections in the coupling constant at the $\Upsilon(4 S)$ resonance looks even more important than at LEP energies.

\section{Results in $N$-space}

In this section we present our analysis in Mellin space and compare our results with the experimental moments of the $D$-hadron cross section, measured by ALEPH, CLEO and BELLE. The data which we consider are the same as the ones which were analysed in the previous section in $x$-space. It was advocated in refs. [24, 39] that working in $N$-space can be theoretically preferable, as one does not need any explicit form for the non-perturbative 


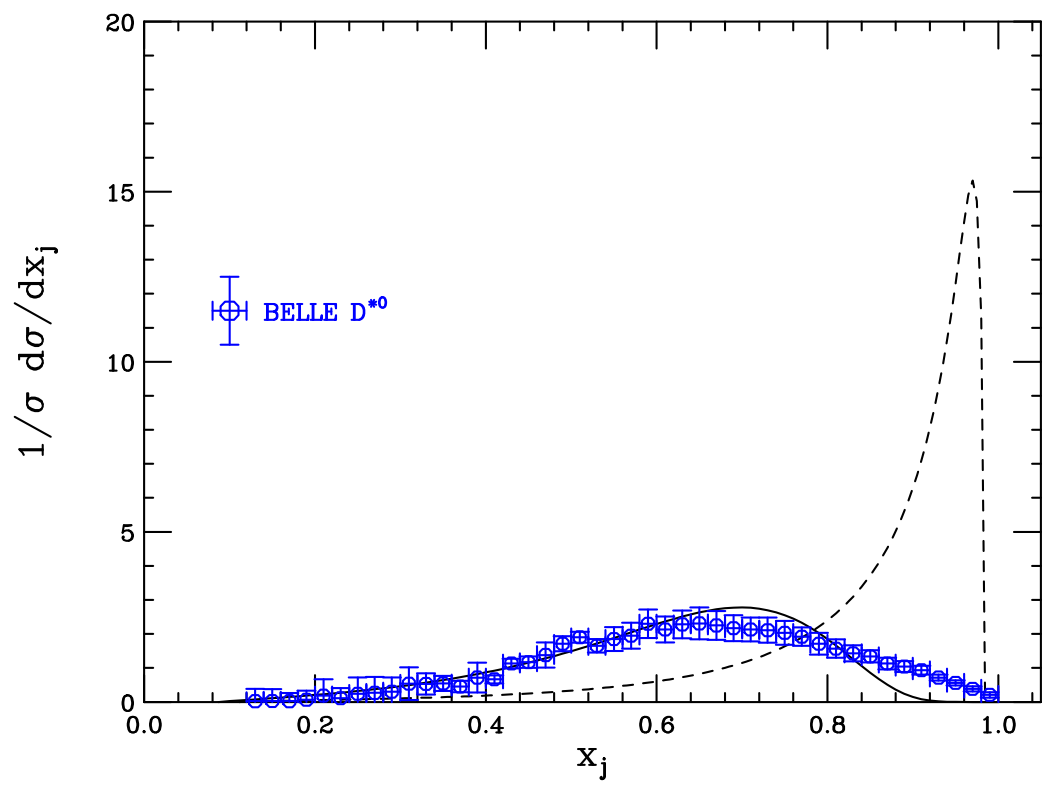

Figure 6: The solid line is our best prediction for charmed-hadron production at the $\Upsilon(4 S)$ resonance $(j=D)$; the dashed line is the purely perturbative $c$-quark spectrum $(j=c)$ yielded by the computation in [11]. Also presented are the BELLE data on the $D^{* 0}$ spectrum.

fragmentation function and its moments can be fitted directly from the data. Of course, this issue does not apply to our case, since we are not fitting any parameter, but nonetheless it is still worthwhile to compare our results with the experimental moments. The $N$-space investigation will be particularly interesting at the $\Upsilon(4 S)$ resonance, where the $x$-space analysis has exhibited quite serious discrepancies.

We calculate the moments of the $D$-hadron cross section directly from the $N$-space formulas, i.e. eq. (4.2), and vary masses and scales as $x$-space, for the sake of estimating the theoretical uncertainty. The experimental moments are the same as the ones presented in ref. [30], with the effect of electromagnetic initial-state radiation subtracted off. We just rescale them so that the first moment of all data sets reads $\sigma_{N=1}=1$, as happens for our theoretical results. Following [1, we first evaluate the uncertainties on the moments due to to the variation of $\mu_{F}, \mu_{0 F}, m_{c}$ and $\alpha_{S}\left(m_{Z}^{2}\right)$ separately, and then estimate the overall theoretical error summing in quadrature all individual uncertainties (see table 2 in ref. (4).

The results of the comparison with the experimental moments are finally presented in figures 7 10, where we investigate how the prediction yielded by our model fares with respect to the moments of ALEPH $D^{*+}$ (figure 7), CLEO and BELLE $D^{0}$ (figure 8), $D^{* 0}$ (figure 9) and $D^{*+}$ (figure 10 ) data. Since our model does not distinguish spin and electric charge, we shall always have the same theoretical moments, regardless of the kind of mesons we are comparing with. As found out in the $x$-space analysis, our predictions are affected by fairly large uncertainties; it is nonetheless interesting that, within the errors, the moments obtained using the resummed calculation provided with the effective-coupling model are compatible with the experimental ones. This result is especially remarkable for the comparison with the data collected at the $\Upsilon(4 S)$ resonance (see figures 8 10), which 


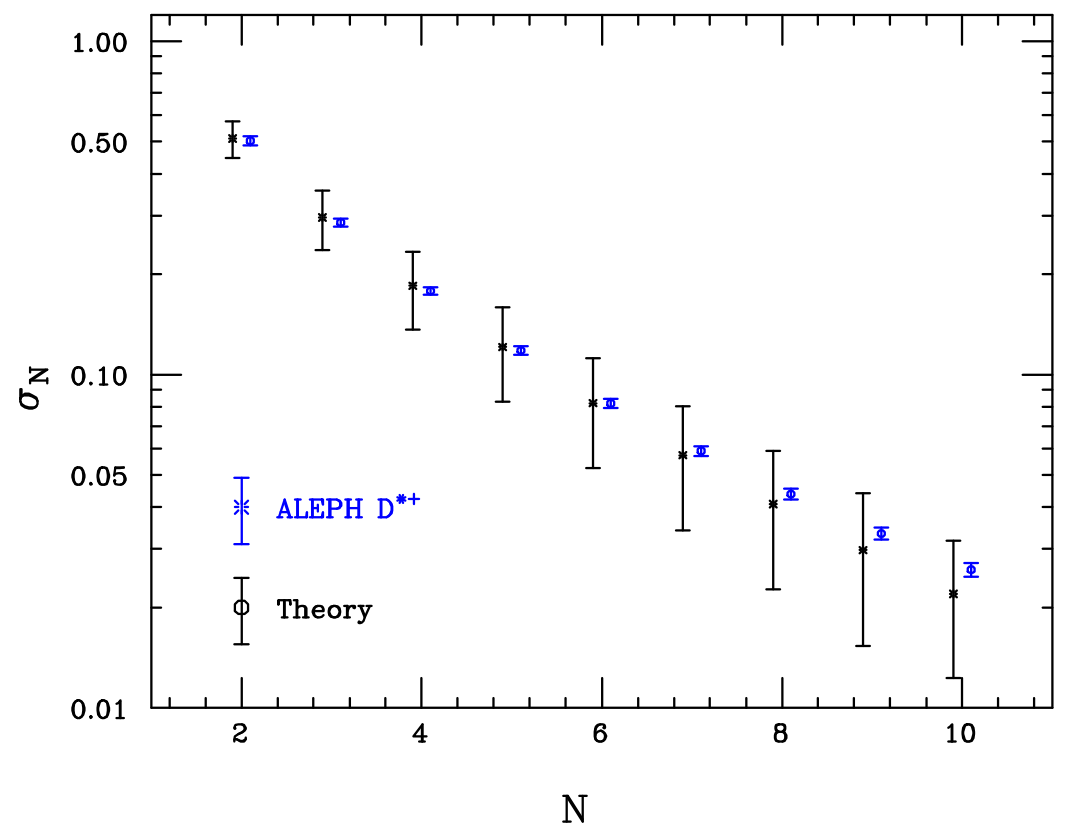

Figure 7: Moments of charmed-hadron cross section according to our effective-coupling model (denoted by 'Theory'), compared with the moments of $D^{*+}$ production at ALEPH. The theoretical errors are estimated by varying the parameters entering in the perturbative calculation, as discussed throughout the text.

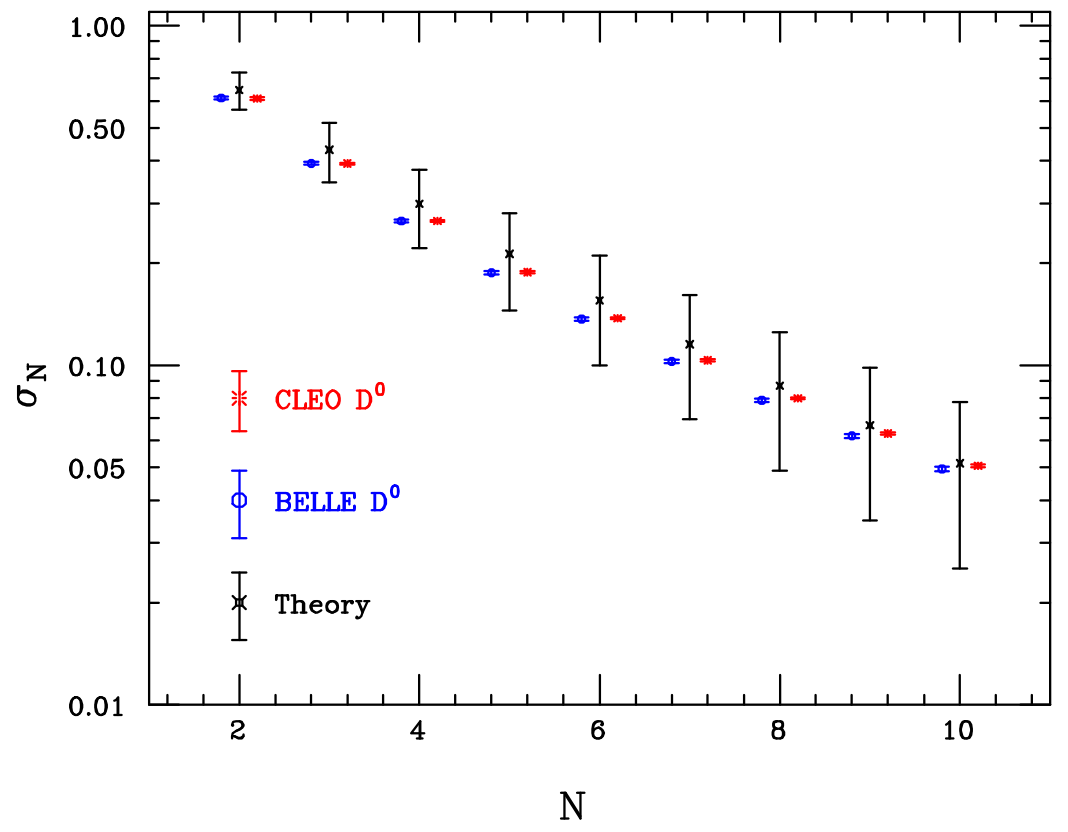

Figure 8: As in figure 7 , but comparing our prediction with the moments of the $D^{0}$ production cross section, measured by the BELLE and CLEO collaborations.

exhibited instead relevant discrepancies in $x$-space. In fact, considering, e.g., the $x$-space $D^{0}$ spectra in figure 3, our model tends to underestimate the event fraction at small and 


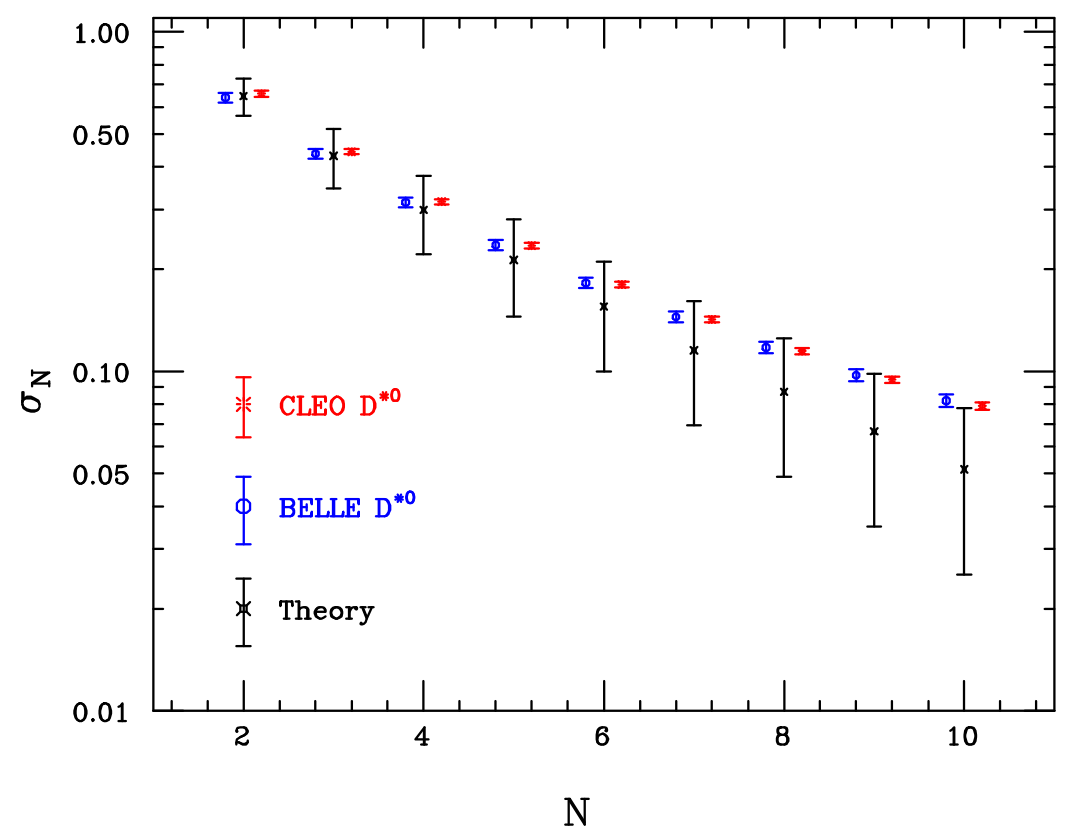

Figure 9: As in figures 7 and 8, with our results faring against the $N$-space $D^{* 0}$ data from CLEO and BELLE.

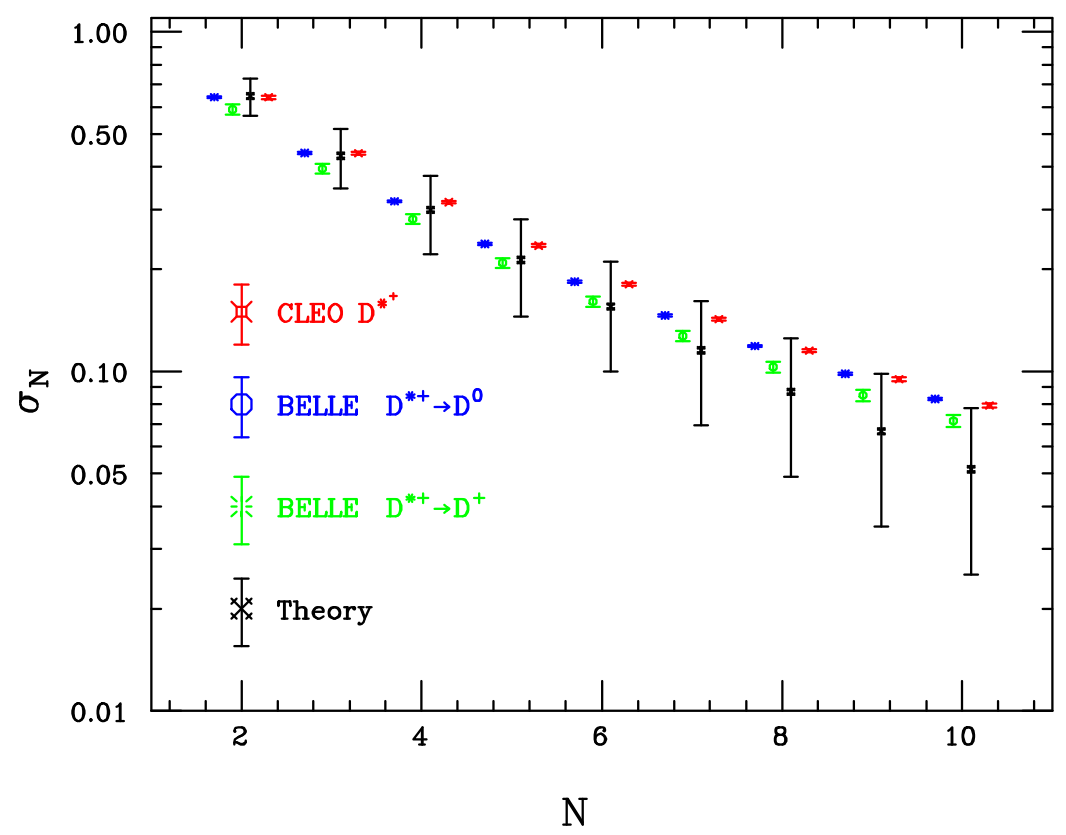

Figure 10: Comparison of our theoretical prediction with the $N$-space $D^{*+}$ data from CLEO and BELLE. As in the $x$-space analysis, we plot separately the $D^{*+}$ moments at BELLE, according to whether they decay via $D^{*+} \rightarrow D^{0}$ or $D^{*+} \rightarrow D^{+}$.

very large $x_{D}$ and overestimate the differential cross section for $0.6 \lesssim x_{D} \lesssim 0.8$. Therefore, when evaluating integrated quantities like the moments, such effects get compensated and one is able to obtain a reasonable description of all $N$-space data (figure 3). A similar result 
was indeed found in [36], where parton showers and resummed calculations were used to describe $B$-hadron production at the $Z^{0}$ pole. The spectrum yielded by the HERWIG Monte Carlo generator [37] gave rise to a quite large $\chi^{2} /$ dof when comparing with $b$ fragmentation data in $x$-space, even after tuning a few parameters. However, HERWIG was able to reproduce fairly well the first few experimental moments of the $B$ cross section.

Referring, e.g., to the average value $\left\langle x_{D}\right\rangle$ of the $D^{0}$ spectra at $B$-factories, corresponding to $\sigma_{N=2}$, the experimental data yield $\left\langle x_{D}\right\rangle=0.610 \pm 0.005$ (CLEO) and $0.612 \pm 0.006$ (BELLE). Using our default perturbative parametrization and effective-coupling model, we obtain instead $\left\langle x_{D}\right\rangle_{\mathrm{th}}=0.647$, above the experimental result. Different choices of the parameters lead nevertheless to even larger or lower estimates of $\sigma_{N=2}$. For example, setting $m_{c}=2.1 \mathrm{GeV}$ and the other parameters to their default values, we obtain $\left\langle x_{D}\right\rangle_{\text {th }}=0.686$, while for $m_{c}=1.5 \mathrm{GeV}$ the second moment reads $\left\langle x_{D}\right\rangle_{\text {th }}=0.599$, below the CLEO and BELLE data. In any case, as already pointed out for the purpose of the $x$-space analysis, a complete NNLO/NNLL should possibly decrease the theoretical error in Mellin space as well. Moreover, since even the best fits in $x$-space were obtained discarding the data points

at $x_{D}>1-\Lambda / m_{c}$, we expect that the comparison with the experimental moments should eventually get worse for very large values of $N$, dominated by the $x_{D} \gtrsim 0.85$ region in $x$ space, where our predictions are systematically below the experimental data. A hint for such a behaviour can be learned from figures 9 and 10, where already the $\sigma_{N=10}$ theoretical moment is only marginally consistent with the data, even within the uncertainties.

\section{Conclusions}

We studied charm-quark fragmentation in $e^{+} e^{-}$annihilation and used a recently proposed model, based on an effective strong coupling constant, as the only source of nonperturbative effects. Such a model was already employed in [3, 4] and gave a reasonable description of $b$-quark fragmentation in $e^{+} e^{-}$annihilation and some $B$-meson decay data. We described charm-quark perturbative production following the perturbative fragmentation approach, with NLO coefficient function and initial condition of the perturbative fragmentation function, NLL DGLAP non-singlet evolution and NNLL large- $x$ resummation. Resummed expressions were matched to the exact NLO ones using the so-called $\ln R$-prescription, which turned out to significantly improve the spectrum near the $x=1$ endpoint. The effective coupling was implemented in the NNLO approximation, as in refs. 迥, 四.

We compared the predictions of our model with data from ALEPH, BELLE and CLEO, corrected for initial-state photon-radiation effects as in [30]. Throughout our analysis, since our non-perturbative model has no tunable parameter, we varied the quantities in the perturbative calculation within typical ranges, according to the values quoted in [34].

We found that our model is able to acceptably describe, for $x_{D}<1-\Lambda / m_{c}$ and within the theoretical and experimental errors, the $D^{*+}$ spectrum from ALEPH. In particular, the best fits to the data are obtained, within our chosen ranges, if we set the factorization scale entering in the initial condition to $\mu_{0 F}=2 m_{c}$. A value of $m_{c}$ consistent with the charm pole mass, rather than the $D$-meson mass, improves the comparison at small $x_{D}$ 
and around the peak. Significant discrepancies were instead found with respect to the $D^{0}, D^{* 0}$ and $D^{*+}$ data from $B$-factories, where we succeeded in obtaining $\chi^{2} /$ dof $\simeq 1$ only when comparing with the BELLE $D^{* 0}$ spectrum, affected by pretty large errors. The experimental data on $D^{0}$ and $D^{*+}$ production at CLEO and BELLE and on $D^{* 0}$ at CLEO exhibit instead very small errors and we did not manage to obtain a reasonable $\chi^{2} /$ dof, even within the theory error. We just noticed that setting $m_{c}=1.5 \mathrm{GeV}$ gives a good description of the data for $x_{D}<0.6$, but some major disagreement is still present for larger values of $x_{D}$. In Mellin space, however, within the fairly large theoretical uncertainties, we managed to reproduce the first ten moments of all considered data samples. We expect nonetheless that for larger values of $N$ some discrepancy should appear, consistently with the observation that even the best $x$-space fits were obtained discarding few large- $x_{D}$ data points.

Anyway, we remind that some problems with reproducing both ALEPH and $B$-factory data were already encountered in ref. [30], where the authors employed a NLO/NLL calculation, a non-perturbative fragmentation function with three free parameters and rescaled $N$ according to eq. (4.5). The hadronization model was tuned to reproduce fairly well all $B$-factory data on neutral as well as charged $D$ - and $D^{*}$-meson production. Nevertheless, the best-fit parametrization did not succeed in reproducing the ALEPH $D^{*+}$ spectrum after evolving to LEP energies. The conclusion of the analysis carried out in [30] was that, in order to reconcile both LEP and $B$-factory data, it was necessary to include power corrections in the process-dependent coefficient function, depending on the process hard scale. This way, one should be able to describe all data, still using the same perturbative accuracy and the same functional form for the non-perturbative part. However, due to the errors in the intermediated $x_{D}$ region, ref. [30] was not able to discriminate whether the missing power corrections should behave according to a $1 / Q$ or a $1 / Q^{2}$ power law, with $Q$ being the centre-of mass energy. In any case, being $m_{Z}$ much larger than $m_{\Upsilon(4 S)}$, one should expect that such a power correction should mainly modify the spectra at the $\Upsilon(4 S)$ resonance, in such a way that the fits of the non-perturbative fragmentation functions at the $Z^{0}$ pole, presented in [30], should eventually work even at $B$-factory energies, after minimal adjustments [38].

As far as our work is concerned, we do find it interesting that, although within the theoretical and experimental errors and after discarding few data points at very large $x_{D}$, our parameter-free model yields $\chi^{2} /$ dof $\simeq 1$ from the comparison with ALEPH $D^{*+}$ data and reproduces the moments of all analysed data sets. The discrepancies of our prediction with respect to the very precise data from CLEO and BELLE in $x$-space clearly deserve further investigation. The results in this paper, along with the ones reported in [4, seem to indicate that the model works better for heavy-quark fragmentation at the $Z^{0}$ pole, while more serious problems show up once the hard scale is lowered. However, only a power correction, such as the one understood in [30], mostly relevant at large $x$ or $N$, may not to be enough to solve the discrepancy with the $B$-factory data, as figures $3 / 5$ show disagreement even around the peak and at small $x_{D}$.

The theoretical uncertainty is expected to decrease after the inclusion of NNLO coefficient functions [12, 13], initial condition [14, 16] and non-singlet splitting functions [15], 
which will also promote DGLAP evolution to NNLL accuracy in the non-singlet sector. Since within our approach we are including power corrections in an effective coupling, any perturbative improvement, such as accounting for $\mathcal{O}\left(\tilde{\alpha}_{S}^{2}\right)$ contributions, will necessarily imply the inclusion of non-perturbative corrections as well. Furthermore, the change (3.12) in the coefficient $A^{(3)}$ has been implemented in the threshold NNLL expressions, but not yet in the splitting functions, whose NNLO corrections do contain a contribution $\sim A^{(3)}$ 15. Including such a term in the splitting functions, along with the redefinition $A^{(3)} \rightarrow \tilde{A}^{(3)}$, may shift the $x_{D}$ spectrum and possibly improve the comparison with the $B$-factory data, as found in [4] for $B$-hadron production at LEP and SLD. We should also expect a relevant impact on our analysis of the possible inclusion of large- $x$ next-to-next-to-next-to-leading logarithmic (NNNLL) terms, whose coefficients have been denoted by $A^{(4)}, B^{(3)}$ and $D^{(3)}$ in Eqs. (2.7) and (2.11). All such coefficients will be modified in a fashion analogous to eq. (3.12) when using $\tilde{\alpha}_{S}\left(k^{2}\right)$. Moreover, the implementation of higher-order threshold contributions in the exponents (2.7) and (2.11) will also lead to the inclusion of further power corrections since, as part of our model, we performed the Mellin transforms exactly.

The other guideline to obtain better agreement with the $x$-space data consists in modifying the effective-coupling model, e.g., introducing a correcting term $\delta \tilde{\alpha}_{S}\left(k^{2}\right)$ as in eq. (3.14), possibly containing extra parameters. In order to accommodate both $D$ and $D^{*}$ data, $\delta \tilde{\alpha}_{S}\left(k^{2}\right)$ may possibly depend on the spin of the considered hadron. However, before speculating about its functional form, we believe that we still need a NNLO/NNLL calculation to reduce the theoretical uncertainty and deal with a more stable prediction. In fact, without a NNLO/NNLL analysis, function $\delta \tilde{\alpha}_{S}\left(k^{2}\right)$ will largely depend on the values chosen for the perturbative parameters and considerably vary according to whether, e.g., one sets $m_{c}=1.5$ or $2.1 \mathrm{GeV}, \mu_{0 F}=m_{c} / 2$ or $2 m_{c}$, and so on.

The large- $x_{D}$ behaviour of our spectra may be improved as well, since even the best comparisons with the data were obtained in this paper for $x_{D}<1-\Lambda / m_{c}$ and in [4] for $x_{B}<1-\Lambda / m_{b}$. An option could be the prescription (4.5) suggested in [30]; in fact, any modification at large $x_{D}$ will indirectly affect, via normalization, the energy distribution at smaller $x_{D}$ as well. Nevertheless, once again, given the uncertainties exhibited by our predictions even at large $x_{D}$, this investigation should be better performed using a calculation of higher accuracy.

Ideally, once the above issues are clarified, one may think of using our model to describe $D$ - and $B$-hadron production at the Tevatron accelerator, along the lines of refs. [39, 40], and extend the results to LHC energies. Nevertheless, unlike the standard analyses, where a non-perturbative fragmentation function is fitted to $e^{+} e^{-}$data and then used in the hadron-collider environment, we are not tuning any parameter to the $e^{+} e^{-}$data. Therefore, possible studies at hadron colliders will be independent checks of the capability of our model to reproduce heavy-quark fragmentation data.

Moreover, we can use the NLO perturbative calculations in [41, 42], along with the effective coupling constant, to predict bottomed-hadron spectra in top $(t \rightarrow b W)$ or Higgs $(H \rightarrow b \bar{b})$ decays at the Tevatron and LHC. Finally, the $c$-fragmentation results here presented can be compared with the ones yielded by Monte Carlo generators, extending the analysis carried out in [36], where parton shower algorithms and resummations were com- 
pared for the purpose of $B$-hadron production in $e^{+} e^{-}$annihilation, top and Higgs decays. For such a comparison to be consistent, however, even the HERWIG [37] and PYTHIA [43] generators will have to be tuned to the same LEP and $B$-factory data analysed throughout this paper. It will also be very interesting to implement the effective coupling constant to replace, e.g., the cluster model [44] which simulates the hadronization in HERWIG and investigate how the Monte Carlo results fare with respect to the experimental data on $D$ and $B$-hadron production. This is in progress as well.

Acknowledgements. We are indebted to U. Aglietti for a series of very useful discussions on the effective-coupling model. We acknowledge D. De Florian for discussions on resummed calculations and M. Cacciari for many conversations on the perturbative fragmentation approach and for providing us with the computing code to obtain the results of ref. 11] presented in figures 2 and 6. We thank C. Oleari for proving us with the data presented in ref. [30], accounting for initial-state radiation effects. This work was partially supported by ALFA-EC funds in the framework of Program HELEN (High Energy Physics Latinoamerican-European Network). G.F. acknowledges support by the European Community's Marie-Curie Research Training Network Programme under contract MRTNCT-2006-035505 "Tools and Precision Calculations for Physics Discoveries at Colliders" G.F. is also grateful to the Physics Department of the University of Buenos Aires for warm hospitality during some of this work.

\section{References}

[1] V.G. Kartvelishvili, A.K. Likehoded and V.A. Petrov, On the fragmentation functions of heavy quarks into hadrons, Phys. Lett. B 78 (1978) 615.

[2] C. Peterson, D. Schlatter, I. Schmitt and P.M. Zerwas, Scaling violations in inclusive $e^{+} e^{-}$ annihilation spectra, Phys. Rev. D 27 (1983) 105.

[3] U. Aglietti, G. Ferrera and G. Ricciardi, Semi-inclusive B decays and a model for soft-gluon effects, Nucl. Phys. B 768 (2007) 85.

[4] U. Aglietti, G. Corcella and G. Ferrera, Modelling non-perturbative corrections to bottom-quark fragmentation, Nucl. Phys. B 775 (2007) 162.

[5] D. Shirkov, Nonpower expansions for QCD observables at low energies, Nucl. Phys. 152 (Proc. Suppl.) (2006) 51.

[6] N. G. Stefanis, W. Schroers and H. C. Kim, Pion form-factors with improved infrared factorization, Phys. Lett. B 449 (1999) 299;

N. G. Stefanis, W. Schroers and H. C. Kim, Analytic coupling and Sudakov effects in exclusive processes: pion and $\gamma^{*} \gamma \rightarrow \pi^{0}$ form factors, Eur. Phys. J. C 18 (2000) 137.

[7] B. Mele and P. Nason, The fragmentation function for heavy quarks in QCD, Nucl. Phys. B 361 (1991) 626.

[8] G. Altarelli, R.K. Ellis, G. Martinelli and S.-Y. Pi, Processes involving fragmentation functions beyond the leading order in QCD, Nucl. Phys. B 160 (1979) 301. 
[9] V.N. Gribov and L.N. Lipatov, Deep inelastic ep scattering in perturbation theory, Sov. J. Nucl. Phys. 15 (1972) 438;

L.N. Lipatov, The parton model and perturbation theory, Sov. J. Nucl. Phys. 20 (1975) 95;

Yu.L. Dokshitzer, Calculation of the structure functions for deep inelastic scattering and $e^{+} e^{-}$annihilation by perturbation theory in quantum chromodynamics (in Russian), Sov. Phys. JETP 46 (1977) 298.

[10] G. Altarelli and G. Parisi, Asymptotic freedom in parton language, Nucl. Phys. B 126 (1977) 298 .

[11] M. Cacciari and S. Catani, Soft-gluon resummation for the fragmentation of light and heavy quarks at large $x$, Nucl. Phys. B 617 (2001) 253.

[12] P.J. Rijken and W.L. van Neerven, Higher-order QCD corrections to the transverse and longitudinal fragmentation functions in electron-positron annihilation, Nucl. Phys. B 487 (1997) 233.

[13] A.D. Mitov and S.O. Moch, QCD corrections to semi-inclusive hadron production in electron positron annihilation at two loops, Nucl. Phys. B 751 (2006) 18.

[14] K. Melnikov and A.D. Mitov, Perturbative heavy quark fragmentation function through $\mathcal{O}\left(\alpha_{S}^{2}\right)$, Phys. Rev. D 70 (2004) 034027.

[15] A.D. Mitov, S. Moch and A. Vogt, Next-to-next-to-leading order evolution of non-singlet fragmentation functions, Phys. Lett. B 638 (2006) 61.

[16] A.D. Mitov, Perturbative heavy quark fragmentation function through $\mathcal{O}\left(\alpha_{S}^{2}\right)$ : Gluon initiated contribution, Phys. Rev. D 71 (2005) 054021.

[17] G. Sterman, Summation of large corrections to short distance hadronic cross- sections, Nucl. Phys. B 281 (1987) 310.

[18] S. Catani and L. Trentadue, Resummation of the QCD perturbative series for hard processes, Nucl. Phys. B 327 (1989) 323.

[19] S. Moch, J.A.M. Vermaseren and A. Vogt, The three loop splitting functions in QCD: the nonsinglet case, Nucl. Phys. B 688 (2004) 101.

[20] S. Moch, J.A.M. Vermaseren and A. Vogt, Nonsinglet structure functions at three loops: fermionic contributions, Nucl. Phys. B 646 (2002) 181.

[21] G.P. Korchemsky and G. Marchesini, Structure function for large $x$ and renormalization of Wilson loop, Nucl. Phys. B 406 (1993) 225.

[22] E. Gardi, On the quark distribution in an on-shell heavy quark and its all-order relations with the perturbative fragmentation function, J. High Energy Phys. 0502 (2005) 053.

[23] S. Catani, G. Turnock, B.R. Webber and L. Trentadue, Thrust distribution in $e^{+} e^{-}$ annihilation, Phys. Lett. B 263 (1991) 491.

[24] M. Cacciari and E. Gardi, Heavy quark fragmentation, Nucl. Phys. B 664 (2003) 299.

[25] S. Catani, D. de Florian, M. Grazzini and P. Nason, Soft gluon resummation for Higgs boson production at hadron colliders, J. High Energy Phys. 07 (2003) 028.

[26] U. Aglietti and G. Ricciardi, A model for next-to-leading order threshold resummed form-factors, Phys. Rev. D 70 (2004) 114008. 
[27] M. Beneke and V.M. Braun, Power corrections and renormalons in Drell-Yan production, Nucl. Phys. B 454 (1995) 253.

[28] D. Amati, A. Bassetto, M. Ciafaloni, G. Marchesini and G. Veneziano, A treatment of hard processes sensitive to the infrared structure of QCD, Nucl. Phys. B 173 (1980) 429.

[29] S. Catani, M.L. Mangano, P. Nason and L. Trentadue, The resummation of soft gluons in hadronic collisions, Nucl. Phys. B 478 (1996) 273.

[30] M. Cacciari, P. Nason and C. Oleari, A study of heavy flavoured meson fragmentation functions in $e^{+} e^{-}$annihilation, J. High Energy Phys. 0604 (2006) 006.

[31] ALEPH collaboration, R. Barate et al., Study of charm production in Z decays, Eur. Phys. J. C 16 (2000) 597.

[32] CLEO collaboration, M. Artuso et al., Charm meson spectra in $e^{+} e^{-}$annihilation at 10.5 GeV c.m.e., Phys. Rev. D 70 (2004) 112001.

[33] BELLE collaboration, R. Seuster et al., Charm hadrons from fragmentation and $B$ decays in $e^{+} e^{-}$annihilation at $\sqrt{s}=10.6$ GeV, Phys. Rev. D 73 (2006) 032002.

[34] Particle Data Group collaboration, W. M. Yao et al., Review of Particle Physics, J. Phys. G $33(2006) 1$.

[35] M. Cacciari, P. Nason and C. Oleari, Crossing heavy-flavour thresholds in fragmentation function, J. High Energy Phys. 0510 (2005) 034.

[36] G. Corcella and V. Drollinger, Bottom-quark fragmentation: Comparing results from tuned event generators and resummed calculations Nucl. Phys. B 730 (2005) 82.

[37] G. Corcella, I.G. Knowles, G. Marchesini, S. Moretti, K. Odagiri, P. Richardson, M.H. Seymour, B.R. Webber, HERWIG 6: An event generator for hadron emission reactions with interfering gluons (including supersymmetric processes), J. High Energy Phys. 0101 (2001) 010 .

[38] M. Cacciari, private communication.

[39] M. Cacciari and P. Nason, Charm cross sections for the Tevatron Run II, J. High Energy Phys. 0309 (2003) 006.

[40] M. Cacciari, S. Frixione, M. L. Mangano, P. Nason and G. Ridolfi, $Q C D$ analysis of first B cross-section data at $1.96 \mathrm{TeV}$, J. High Energy Phys. 0407 (2004) 033.

[41] M. Cacciari, G. Corcella and A.D. Mitov, Soft-gluon resummation for bottom fragmentation in top quark decay, J. High Energy Phys. 0212 (2002) 015.

[42] G. Corcella, Fragmentation in $H \rightarrow b \bar{b}$ processes, Nucl. Phys. B 705 (2005) 363, Erratum ibid. 713 (2005) 609.

[43] T. Sjostrand, S. Mrenna and P. Skands, PYTHIA 6.4 physics and manual, J. High Energy Phys. 0605 (2006) 026.

[44] B.R. Webber, A QCD model for jet fragmentation including soft gluon interference, Nucl. Phys. B 238 (1984) 492. 